\title{
Measure Properties of Game Tree Languages
}

\author{
Tomasz Gogacz ${ }^{1}$, Henryk Michalewski ${ }^{2}$, Matteo Mio $^{3}$, and Michał Skrzypczak ${ }^{2}$ \\ 1 Institute of Computer Science, University of Wrocław, Poland \\ 2 Faculty of Mathematics, Informatics and Mechanics, University of Warsaw, Poland \\ 3 Computer Laboratory, University of Cambridge, England (UK)
}

\begin{abstract}
We introduce a general method for proving measurability of topologically complex sets by establishing a correspondence between the notion of game tree languages from automata theory and the $\sigma$-algebra of $\mathcal{R}$-sets, introduced by A. Kolmogorov as a foundation for measure theory. We apply the method to answer positively to an open problem regarding the game interpretation of the probabilistic $\mu$-calculus.
\end{abstract}

\section{Introduction}

Among logics for expressing properties of nondeterministic (including concurrent) processes, represented as transition systems, Kozen's modal $\mu$-calculus [15] plays a fundamental rôle. This logic enjoys an intimate connection with parity games, which offers an intuitive reading of fixed-points, and underpins the existing technology for model-checking $\mu$-calculus properties. An abstract setting for investigating parity games, using the tools of descriptive set theory, is given by so-called game tree languages (see, e.g. [2]). The language $\mathcal{W}_{i, k}$ is the set of parity games with priorities in $\{i \ldots k\}$, played on an infinite binary tree structure, which are winning for Player $\exists$. The $(i, k)$-indexed sets $\mathcal{W}_{i, k}$ form a strict hierarchy of increasing topological complexity called the index hierarchy of game tree languages (see $[5,1,2])$. Precise definitions are presented in Section 2.

For many purposes in computer science, it is useful to add probability to the computational model, leading to the notion of probabilistic nondeterministic transition systems (PNTS's). In an attempt to identify a satisfactory analogue of Kozen's $\mu$-calculus for expressing properties of PNTS's, the third author has recently introduced in $[19,20]$ a quantitative fixed-point logic called probabilistic $\mu$-calculus with independent product $(\mathrm{pL} \mu)$. A central contribution of [20] is the definition of a game interpretation of $\mathrm{pL} \mu$, given in terms of a novel class of games generalizing ordinary two-player stochastic parity games. While in ordinary twoplayer (stochastic) parity games the outcomes are infinite sequences of gamestates, in $\mathrm{pL} \mu$-games the outcomes are infinite trees, called branching plays, whose vertices are labelled with game-states. This is because in $\mathrm{pL} \mu$ some of the game-states, called branching states, are interpreted as generating distinct game-threads, one for each successor state of the branching state, which continue their execution concurrently and independently. The winning set of a $\mathrm{pL} \mu$-game is therefore a collection of branching plays specified by a combinatorial condition associated with the structure of the game arena. 
Unlike winning sets of ordinary two-player (stochastic) parity games, which are well-known to be Borel sets, the winning sets of $\mathrm{pL} \mu$-games generally belong to the $\boldsymbol{\Delta}_{2}^{1}$-class of sets in the projective hierarchy of Polish spaces [20, Theorem 4.20]. This high topological complexity is a serious concern because $\mathrm{pL} \mu$-games are stochastic, i.e. the final outcome (the branching play) is determined not only by the choices of the two players but also by the randomized choices made by a probabilistic agent. A pair of strategies for $\exists$ and $\forall$, representing a play up-to the choice of the probabilistic agent, only defines a probability measure on the space of outcomes. For this reason, one is interested in the probability of a play to satisfy the winning condition. Under the standard Kolmogorov's measure-theoretic approach to probability theory, a set has a welldefined probability only if it is a measurable set ${ }^{4}$. Due to a result of Kurt Gödel (see $[11, \S 25]$ ), it is consistent with Zermelo-Fraenkel Set Theory with the Axiom of Choice (ZFC) that there exists a $\boldsymbol{\Delta}_{2}^{1}$ set which is not measurable. This means that it is not possible to prove (in ZFC) that all $\boldsymbol{\Delta}_{2}^{1}$-sets are measurable. However it may be possible to prove that a particular set (or family of sets) in the $\boldsymbol{\Delta}_{2}^{1}$-class is measurable. In [19] the author asks the following question ${ }^{5}$ :

Question: are the winning sets of $\mathrm{pL} \mu$-games provably measurable?

This problem provided the original motivation of our work. We will answer positively to the question by developing a general method for proving measurability of topologically complicated sets.

This type of questions has been investigated since the first developments of measure theory, in late 19th century, as the existence of non-measurable sets (e.g. Vitali sets [11]) was already known. The measure-theoretic foundations of probability theory are based around the concept of a $\sigma$-algebra of measurable events on a space of potential outcomes. Typically, the $\sigma$-algebra is assumed to contain all open sets. Hence the minimal $\sigma$-algebra under consideration consists of all Borel sets whereas the maximal consists, by definition, of the collection of all measurable sets. The Borel $\sigma$-algebra, while simple to work with, lacks important classes of measurable sets (e.g. $\Pi_{1}^{1}$-complete sets). On the other hand, the full $\sigma$-algebra of measurable sets may be difficult to work with since there is no constructive methodology for establishing its membership relation, i.e. for proving that a given set belongs to this $\sigma$-algebra.

This picture led to a number of attempts to find the largest $\sigma$-algebra, extending the Borel $\sigma$-algebra and including as many measurable sets as possible and, at the same time, providing practical techniques for establishing the membership relation. A general methodology for constructing such $\sigma$-algebras is to identify a family $\mathcal{F}$ of safe operations on sets which, when applied to measurable sets are guaranteed to produce measurable sets. When the operations considered have countable arity (e.g. countable union), the $\sigma$-algebra generated by the open sets closing under the operations in $\mathcal{F}$ admits a transfinite decomposition into

\footnotetext{
${ }^{4}$ More precisely, universally measurable, see Section 2.

${ }^{5}$ Statement "is $\mathrm{mG}-\mathrm{UM}\left(\Gamma_{p}\right)$ true?", see Definition 5.1.18 and discussion at the end of Section 4.5 in [19]. See also Section 8.1 in [20].
} 
$\omega_{1}$ levels, and this allows the membership relation to be established inductively. The simplest case is given by the $\sigma$-algebra of Borel sets, with $\mathcal{F}$ consisting of the operations of complementation and countable union. Other less familiar examples include $\mathcal{C}$-sets studied by E. Selivanovski [21], Borel programmable sets proposed by D. Blackwell [4] and $\mathcal{R}$-sets proposed by A. Kolmogorov [14].

The $\sigma$-algebra of $\mathcal{R}$-sets is, to our knowledge, the largest ever considered. Most measurable sets arising in ordinary mathematics are $\mathcal{R}$-sets belonging to the finite levels of the transfinite hierarchy of $\mathcal{R}$-sets. For example, all Borel sets, analytic sets, co-analytic sets and Selivanovski's $\mathcal{C}$-sets lie in the first two levels [8]. Thus, for most practical purposes, the following principle is valid:

Principle: "all practically useful measurable sets belong to the finite levels of the transfinite hierarchy of Kolomogorov's $\mathcal{R}$-sets."

Contributions. The definition of $\mathcal{R}$-sets in [14], formulated in terms of operations on sets and transformations on operations (Section 3), is purely settheoretical. As a main technical contribution of this work, we provide an alternative game-theoretical characterization of the finite levels of the hierarchy of $\mathcal{R}$-sets in terms of game tree languages $\mathcal{W}_{i, k}$.

Theorem 1. $\mathcal{W}_{k-1,2 k-1}$ is complete for the $k$-th level of the hierarchy of $\mathcal{R}$-sets.

As a consequence one can establish the measurability of a given set $A \subseteq X$ by constructing a continuous reduction to $\mathcal{W}_{i, k}$. This can be thought as a coding $f$ of elements in $X$ in terms of parity games with priorities in $\{i, \ldots, k\}$ such that $x \in A$ if and only if $f(x)$ is winning for Player $\exists$. Parity games are well-known and relatively simple to work with. Thus the proof method allows for easier applications. Since $\mathcal{R}$-sets exhaust the realm of reasonable measurable sets, and the sets $\mathcal{W}_{i, k}$ are complete among $\mathcal{R}$-sets, the method should cover most cases.

Additionally, in Section 6 , we investigate the special continuity property of measures on $\mathcal{W}_{i, k}$ with respect to the approximations $\mathcal{W}_{i, k}^{\alpha}$, crucially required in the proof of determinacy of pL $\mu$-games of $[20,19]$. As observed in [19], the property follows from the set-theoretic Martin Axiom at $\aleph_{1}\left(\mathrm{MA}_{\aleph_{1}}\right)$. The problem of whether the property holds in ZFC alone is left open in [19]. Our contribution shows that the continuity property holds for $\mathcal{W}_{0,1}$ and that for higher indices it does not depend on cardinality assumptions such as the negation of the Continuum Hypothesis, which is one of the consequences of $\mathrm{MA}_{\aleph_{1}}$.

Applications. As already observed in $[19, \S 5.4]$, the winning sets of $\mathrm{pL} \mu$-games reduce to game tree languages. Thus Theorem 1 settles the question posed in [19] about the measurability of $\mathrm{pL} \mu$ winning sets. More generally, our result can find applications in solving similar problems. For example, in models of probabilistic concurrent computation (e.g. probabilistic Petri nets [16], probabilistic event structures [9], stochastic distributed games [22]), executions are naturally modelled by configurations of event structures (i.e. special kinds of acyclic graphs) and not by sequences. Many natural predicates on executions (e.g. the collection of well-founded graphs) are of high topological complexity. 
Related Work. Beside the original work of Kolmogorov [14], the measure theoretic properties of $\mathcal{R}$-sets are investigated with set-theoretic methods by Lyapunov in [17]. A game-theoretic approach to $\mathcal{R}$-sets, closely related to this work, is developed by Burgess in [8] where the following characterization is stated as a remark without a formal proof: (1) every set $A \subseteq X$ belongs to a finite level of the hierarchy of $\mathcal{R}$-sets if and only if it is of the form $A=\circlearrowright(K)$, for some set $K \subseteq \omega^{\omega}$ which is a Boolean combination of $F_{\sigma}$ sets, and (2) the levels of the hierarchy of $\mathcal{R}$-sets are in correspondence with the levels of the difference hierarchy (see [13, $\S 22 . \mathrm{E}]$ ) of $F_{\sigma}$ sets. The operation $\partial$ is the so-called game quantifier (see [13, $\S 20 . \mathrm{D}]$ and $[6,7,12,18])$. Admittedly, our characterization of $\mathcal{R}$-sets in terms of game tree languages $\mathcal{W}_{i, k}$, can be considered as a modern variant of the result of Burgess. ${ }^{6}$ Having concrete examples of complete sets, however, sheds light on the concept of $\mathcal{R}$-sets and, in analogy with the study of complexity classes in computational complexity theory, may simplify further investigations. Lastly, it is suggestive to think that the origins of the concept of parity games, developed since the 80's in Computer Science to investigate $\omega$-regular properties of transition systems, could be backdated to the original work of A. Kolmogorov.

\section{Basic Notions from Descriptive Set Theory}

We assume the reader is familiar with the basic notions of descriptive set theory and measure theory. We refer to [13] as a standard reference on these subjects.

Given two sets $X$ and $Y$, we denote with $X^{Y}$ the set of functions from $Y$ to $X$. We denote with 2 and $\omega$ the two element set and the set of all natural numbers, respectively. The powerset of $X$ will be denoted by both $2^{X}$ and $\mathcal{P}(X)$, as more convenient to improve readability. A topological space is Polish if it is separable and the topology is induced by a complete metric. A set is clopen if it is both closed and open. A space is zero-dimensional if the clopen subsets form a basis of the topology. In this work we limit our attention to zero-dimensional Polish spaces. Let $X, Y$ be two topological spaces and $A \subseteq X, B \subseteq Y$ be two sets. We say that $A$ is Wadge reducible to $B$, written as $A \leq_{W} B$, if there exists a continuous function $f: X \rightarrow Y$ such that $A=f^{-1}(B)$. Two sets $A$ and $B$ are Wadge equivalent (denoted $A \sim_{W} B$ ) if $A \leq_{W} B$ and $B \leq_{W} A$ hold. Given a family $\mathcal{C}$ of subsets of $X$, we say a set $A \in \mathcal{C}$ is Wadge complete if $B \leq_{W} A$ holds for all $B \in \mathcal{C}$. Given a Polish space $X$, we denote with $\mathcal{M}_{=1}(X)$ the Polish space of all Borel probability measures $\mu$ on $X$ (see e.g. [13, Theorem 17.22]). A set $N \subseteq X$ is $\mu$-null if there exists a Borel set $B \supseteq N$ such that $\mu(B)=0$. A set $A \subseteq X$ is $\mu$-measurable if $A=B \cup N$, for a Borel set $B$ and a $\mu$-null set $N$. A set $A \subseteq X$ is universally measurable if it is $\mu$-measurable for all $\mu \in \mathcal{M}_{=1}(X)$. In what follows we omit the "universally" adjective.

\footnotetext{
${ }^{6}$ The fact that the sets $\mathcal{W}_{i, k}$ are $\mathcal{R}$-sets follows from the above formulation of Burgess' theorem. Also, our Theorem 1 can be easily inferred for $k=1$. The case of $k=2$ follows from the theorem of Burgess in conjunction with [18]. Our proof of Theorem 1 yields an independent and formal argument which backs the above statement of Burgess' theorem.
} 
Given two natural numbers $i<k$, the set $\operatorname{Tr}_{i, k}$ of all complete (i.e. without leaves) binary trees whose vertices are labelled by elements of $\{\exists, \forall\} \times\{i, \ldots, k\}$ is endowed with a Polish topology (see e.g. [2]). Each $t \in \operatorname{Tr}_{i, k}$ can be interpreted as a two-player parity game with priorities in $\{i, \ldots, k\}$, with players $\exists$ and $\forall$ controlling vertices labelled by $\exists$ and $\forall$, respectively.

Definition 1. Given two natural numbers $i<k$, the game tree language $\mathcal{W}_{i, k}$ is the subset of $\operatorname{Tr}_{i, k}$ consisting of all parity games admitting a winning strategy for $\exists$. The pair $(i, k)$ is called the index of $\mathcal{W}_{i, k}$.

Clearly, there is a natural Wadge equivalence between the languages $\mathcal{W}_{i, k}$ and $\mathcal{W}_{i+2, k+2}$. Therefore, we identify indices $(i, k)$ and $(i+2 j, k+2 j)$ for every $i \leq k$ and $j \in \omega$. Indexes can be partially ordered by defining $(i, k) \subseteq\left(i^{\prime}, k^{\prime}\right)$ if and only if $\{i, \ldots, k\} \subseteq\left\{i^{\prime}, \ldots, k^{\prime}\right\}$.

\section{Definition and Basic Properties of $\mathcal{R}$-sets}

Following Kolmogorov, we introduce $\mathcal{R}$-sets as the family generated by the $o p$ eration $\bigcup \circ \bigcap$ and closing under a transformation co- $\mathcal{R}$. It will be convenient to assume that the countably many inputs of an operation $\Gamma$ are indexed by a countable set (called the arena) denoted by $\mathbb{A}_{\Gamma}$. Thus an operation $\Gamma$ has type $\Gamma: \mathcal{P}(X)^{\mathbb{A}_{\Gamma}} \rightarrow \mathcal{P}(X)$. The operations of countable union and intersections are denoted by $\bigcup$ and $\bigcap$, respectively, and their arena is defined as $\mathbb{A}_{\cup}=\mathbb{A}_{\bigcap}=\omega$.

Definition 2. Given two operations $\Gamma$ and $\Theta$ their composition $\Theta \circ \Gamma$ is the operation with arena $\mathbb{A}_{\Gamma} \times \mathbb{A}_{\Theta}$ defined as: $\Theta \circ \Gamma\left(\left\{A_{s, s^{\prime}} \mid s \in \mathbb{A}_{\Gamma}, s^{\prime} \in \mathbb{A}_{\Theta}\right\}\right)=$ $\Theta\left(\left\{\Gamma\left(\left\{A_{s, s^{\prime}} \mid s \in \mathbb{A}_{\Gamma}\right\}\right) \mid s^{\prime} \in \mathbb{A}_{\Theta}\right\}\right)$.

Definition 3. $A$ basis for an operation $\Gamma$ is a set $N_{\Gamma} \subseteq 2^{\mathbb{A}_{\Gamma}}$ such that

$$
\Gamma\left(\left\{A_{s}: s \in \mathbb{A}_{\Gamma}\right\}\right)=\bigcup_{S \in N_{\Gamma}} \bigcap_{s \in S} A_{s}
$$

Not all operations have a basis, but a family $N \subseteq 2^{\mathbb{A}}$ uniquely determines an operation $\Gamma$ with arena $\mathbb{A}$ and basis $N$. In what follows we will only consider operations $\Gamma$ with a basis $N_{\Gamma}$. One can check that $N_{\cap}=\{\{n\} \mid n \in \omega\}$ and $N_{\cap}=\{\omega\}$. A proof is presented in Section B.3.

Definition 4. For a given operation $\Theta$ with arena $\mathbb{A}$ and basis $N_{\Theta}$, we define a dual operation co- $\Theta=\Gamma$ with the same arena $\mathbb{A}$ and basis $N_{\mathrm{co}-} \Theta \stackrel{\text { def }}{=}$ $\left\{S \in 2^{\mathbb{A}} \mid \forall T \in N_{\Theta} T \cap S \neq \emptyset\right\}$. One can notice that equivalently we can define co- $\Theta\left(\left\{A_{s}: s \in \mathbb{A}\right\}\right)=\bigcap_{S \in N} \bigcup_{s \in S} A_{s}$.

As an illustration of this definition, the equalities co- $U=\bigcap$ and co- $\bigcap=\bigcup$ hold. The simple proof is presented in Section B.3. 
Definition 5. The $\mathcal{R}$-transformation of an operation $\Theta$ with basis $N_{\Theta}$ is the operation $\mathcal{R} \Theta=\Gamma$, with arena $\mathbb{A}_{\Gamma}=\left(\mathbb{A}_{\Theta}\right)^{*}$ (finite sequences of elements in $\mathbb{A}_{\Theta}$ ) uniquely determined by the basis:

$$
N_{\mathcal{R} \Theta} \stackrel{\text { def }}{=}\left\{S \subseteq\left(\mathbb{A}_{\Theta}\right)^{*} \mid \exists T \subseteq S . \quad \epsilon \in T \wedge \forall t \in T\left\{v \in \mathbb{A}_{\Theta}: t v \in T\right\} \in N_{\Theta}\right\}
$$

where $\epsilon$ denotes the empty sequence and tv the concatenation of $t \in\left(\mathbb{A}_{\Theta}\right)^{*}$ with



$$
\Theta_{k} \stackrel{\text { def }}{=}(\operatorname{co}-\mathcal{R})^{k}(\bigcup \circ \bigcap) \text {. }
$$

Definition 6. For a positive number $k \geq 1$, we say that a set $A \subseteq X$ is an $\mathcal{R}$-set of $k$-th level if and only if $A=\Theta_{k}\left(\left\{U_{s}: s \in \mathbb{A}_{\Theta_{k}}\right\}\right)$ for some clopen sets $U_{s} \subseteq X$.

In what follows by $\mathcal{R}$-sets we mean $\mathcal{R}$-sets of finite levels. For a proof of the following lemma see Section B.1.

Lemma 1 ([8]). The $k$-th level of $\mathcal{R}$-sets is closed under pre-images of continuous functions.

We say that an operation $\Gamma$ preserves measurability if for any family $\mathcal{E}=$ $\left\{A_{s}\right\}_{s \in \mathbb{A}_{\Gamma}}$ of measurable sets, the set $\Gamma(\mathcal{E})$ is measurable. The following property motivates the notion of $\mathcal{R}$-sets (see Section B.2 in the Appendix for a proof):

Theorem 2 ([17, Theorem 4]). If $\Gamma$ and $\Theta$ preserve measurability then $\Gamma \circ \Theta$, $\mathcal{R} \Gamma$, and co- $\Gamma$ preserve measurability.

Corollary 31. All $\mathcal{R}$-sets are measurable.

\section{Matryoshka games}

In this section we define Matryoshka games, a variant of parity games which make it easier to establish a connection with the operations $\Theta_{k}$ defined in Section 3.

A Matryoshka game is the familiar structure of a two-player parity game played on an infinite countably branching graph, extended with a labelling function assigning to each finite play (i.e. every sequence of game-states ending in a terminal state) a play label. Formally:

$$
\mathcal{G}=\left\{V^{\mathcal{G}}=V_{\exists}^{\mathcal{G}} \sqcup V_{\forall}^{\mathcal{G}}, F^{\mathcal{G}}, E^{\mathcal{G}}, v_{I}^{\mathcal{G}}, \Omega^{\mathcal{G}}, \mathbb{A}^{\mathcal{G}}, \text { label }^{\mathcal{G}}\right\},
$$

such that $\left\{V^{\mathcal{G}}=V_{\exists}^{\mathcal{G}} \sqcup V_{\forall}^{\mathcal{G}}, F^{\mathcal{G}}, E^{\mathcal{G}}, v_{I}^{\mathcal{G}}, \Omega^{\mathcal{G}}\right\}$ is a standard parity game with terminal positions $F^{\mathcal{G}}$, see Section B.5. Additionally, $\mathbb{A}^{\mathcal{G}}$ is a set of play labels, and label ${ }^{\mathcal{G}}:\left(V^{\mathcal{G}}\right)^{*} F^{\mathcal{G}} \rightarrow \mathbb{A}^{\mathcal{G}}$ is a function assigning to finite plays their play labels.

We assume that for every $v \in V^{\mathcal{G}}$ there is at least one $v^{\prime} \in V^{\mathcal{G}} \cup F^{\mathcal{G}}$ such that $\left(v, v^{\prime}\right) \in E^{\mathcal{G}}$, so that the only terminal game-states are in $F^{\mathcal{G}}$. As for standard parity games, the pair $(i, k)$ containing the minimal and maximal values of $\Omega$ is called the index of the game. By $P \in\{\exists, \forall\}$ we denote the players of the game. The opponent of $P$ is denoted by $\bar{P}$. 
A play is defined as usual as a maximal path in the arena, i.e., either as a finite sequence in $\left(V^{\mathcal{G}}\right)^{*} F^{\mathcal{G}}$ or as an infinite sequence $\left(V^{G}\right)^{\mathcal{G}}$. Similarly, a strategy $\sigma$ for a player $P$ is a function $\sigma:\left(V^{\mathcal{G}}\right)^{*} V_{P}^{\mathcal{G}} \rightarrow V^{\mathcal{G}} \cup F^{\mathcal{G}}$ defined as expected.

The novelty in Matryoshka games is given by the set of play labels $\mathbb{A}^{\mathcal{G}}$ and the associated labelling function label ${ }^{\mathcal{G}}$. These are used to define parametric winning condition in the Matryoshka game, as we now describe.

A set of play labels $X \subseteq \mathbb{A}^{\mathcal{G}}$ is called a promise. A finite play $\pi$ is winning for $\exists$ with promise $X$ if label $(\pi) \in X$. An infinite play is $\pi$ is winning for $\exists$ if $\left(\lim \sup _{n \rightarrow \infty} \Omega^{\mathcal{G}}(\pi(n))\right)$ is even, as usual. If a play is not winning for $\exists$ then it is winning for $\forall$. A strategy $\sigma$ for Player $P$ is winning in the Matryoshka game $\mathcal{G}$ with promise $X$ if, for every counter-strategy $\tau$ of $\bar{P}$, the resulting play $\pi(\sigma, \tau)$ is winning for $P$ with promise $X$, in the sense just described. The following proposition directly follows from the well-known determinacy of parity games.

Proposition 1. If $\mathcal{G}$ is a Matryoshka game with play labels $\mathbb{A}^{\mathcal{G}}$ and $X \subseteq \mathbb{A}^{\mathcal{G}}$ then exactly one of the players has a winning strategy in $\mathcal{G}$ with promise $X$.

The point of having parametrized winning conditions in Matryoshka games is the possibility of defining set-theoretical operations with a direct game interpretation. Given a Polish space $X$, the operation on sets (see Section 2) associated with a Matryoshka game $\mathcal{G}$ has arena $\mathbb{A}^{\mathcal{G}}$ and is defined as follows:

$$
\mathcal{G}(\mathcal{E}) \stackrel{\text { def }}{=}\left\{x \in X: \exists \text { has a w. s. in } \mathcal{G} \text { with promise }\left\{s \in \mathbb{A}^{\mathcal{G}}: x \in E_{s}\right\}\right\}
$$

where $\mathcal{E}=\left\{E_{s}: s \in \mathbb{A}^{\mathcal{G}}\right\}$ is a family of subsets of $X$.

We now sketch the definition of a Matryoshka game, called $\mathcal{G}_{0}$, whose associated operation is precisely the operation $(\bigcup \circ \bigcap)$ of Section 2 (a proof is presented in Section B.5). The structure of $\mathcal{G}_{0}$ is depicted in Figure 1. This is a simple game of two steps, where $\exists$ chooses a number $n$ and $\forall$ responds choosing a number $m$. Every play is finite and of the form $\langle\epsilon, n, n . m\rangle$. The set of play labels $\mathbb{A}^{\mathcal{G}_{0}}$ is defined as $\omega \times \omega$ and label ${ }^{\mathcal{G}}(\langle\epsilon, n, n . m\rangle)=(n, m)$.

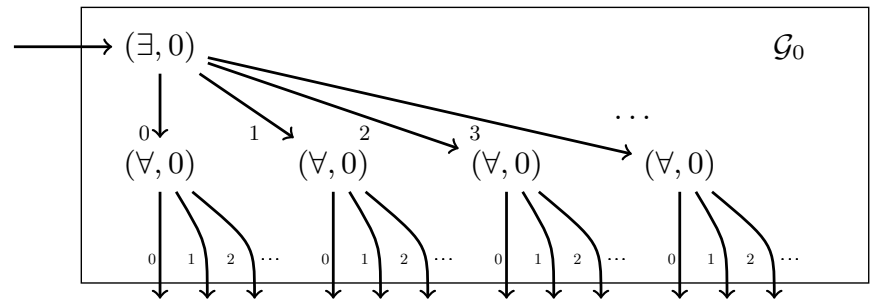

Fig. 1. The game $\mathcal{G}_{0}$ corresponding to the operation $\bigcup \circ \bigcap$.

We now introduce transformations on games which directly match the corresponding transformations on operations defined in Section 2. Due to space limitations, formal introduction of these transformation is given in Section B.5. 
For a Matryoshka game $\mathcal{G}$ of index $(i, k)$, we define co- $\mathcal{G}$ as the game obtained from $\mathcal{G}$ by replacing the sets $V_{\exists} \leftrightarrow V_{\forall}$ and increasing all priorities in $\Omega$ by 1 . Note that the index of co- $\mathcal{G}$ is $(i+1, k+1)$, and that the sets of plays in the two games are equal. We define $\mathbb{A}^{\text {co- } \mathcal{G}} \stackrel{\text { def }}{=} \mathbb{A}^{G}$ and label ${ }^{\text {co- } \mathcal{G}}(\pi) \stackrel{\text { def }}{=}$ label $^{\mathcal{G}}(\pi)$.

Lastly, we define the $\mathcal{R}$ transformation on games. Let us take a Matryoshka game $\mathcal{G}$ of index $(i, k)$. Let $2 j$ be the minimal even number such that $k \leq 2 j$. The game $\mathcal{R G}$ is depicted on Figure 2.

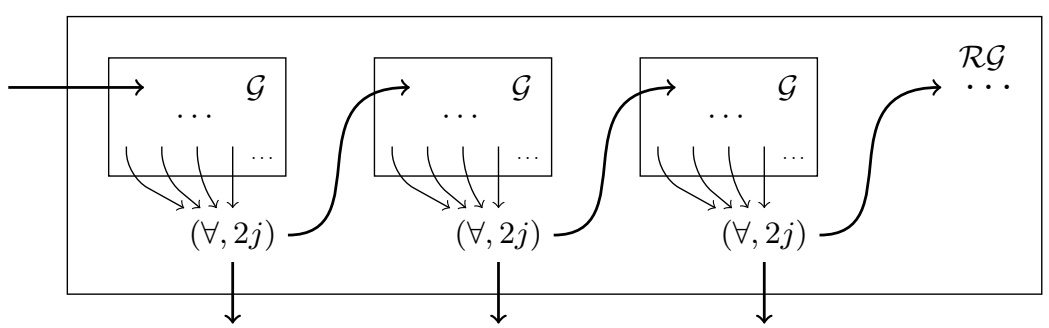

Fig. 2. The game $\mathcal{R G}$.

A play in the game $\mathcal{R G}$ starts from a first copy of $\mathcal{G}$. In this inner game, the play $\pi$ can either be infinite (in which case $\pi$ is a valid play in $\mathcal{R G}$ and is winning for Player $P$ iff it is winning for $P$ in $\mathcal{G}$ ) or terminate in a terminal state of $\mathcal{G}$. In this latter case, Player $\forall$ can either conclude the game $\mathcal{R G}$, or start another session of the inner game $\mathcal{G}$. Observe that if $\forall$ always chooses to start a new session, he loses because the even priority $2 j$ is maximal in $\mathcal{R G}$.

The set of play labels $\mathbb{A}^{\mathcal{R G}}$ is defined as $\left(\mathbb{A}^{\mathcal{G}}\right)^{*}$, i.e., the set of finite sequences of play labels in $\mathcal{G}$. Let $\pi$ be a play in $\mathcal{R G}$ that passes through $n$ copies of $\mathcal{G}$ and then ends in a terminal position of $\mathcal{R G}$. In that case $\pi$ can be decomposed into $n$ plays $\pi_{0}, \ldots, \pi_{n-1}$ in $\mathcal{G}$. We then define the labelling function of $\mathcal{R G}$ as follows:

$$
\operatorname{label}^{\mathcal{R G}}(\pi) \stackrel{\text { def }}{=}\left(\operatorname{label}^{\mathcal{G}}\left(\pi_{0}\right), \text { label }^{\mathcal{G}}\left(\pi_{1}\right), \ldots, \text { label }^{\mathcal{G}}\left(\pi_{n-1}\right)\right) .
$$

Given the basic Matryoshka game $G_{0}$ and the two transformations of games co- and $\mathcal{R}$, we can construct more and more complex "nested" games. This fact motivates the name of this class of games. We denote with $\mathcal{G}_{k}$ the game obtained from $G_{0}$ by iterating $k$-times the composed transformation co- $\mathcal{R}$.

By the definition, the game $\mathcal{G}_{k}$ for $k>0$ consists of infinitely many copies of $\mathcal{G}_{k-1}$ and an additional set of new vertices as depicted on Figure 2. These new vertices are called the $k$-layer of the game. Therefore, by unfolding the definition, each vertex $v$ of $\mathcal{G}_{k}$ is either a vertex of a copy of $\mathcal{G}_{0}$ or it belongs to a $j$-layer for some $1 \leq j \leq k$. Observe that if $v$ is in a $j$-layer of $\mathcal{G}_{k}$ then

$$
\Omega^{\mathcal{G}_{k}}(v)=k+j-1 \quad \text { and } \quad\left(v \in V_{\forall}^{\mathcal{G}_{k}} \Leftrightarrow k+j-1 \equiv 0(\bmod 2)\right) .
$$


We are now ready to state the expected correspondence between the operation $\Theta_{k}$ of Section 2 and the Matryoshka game $\mathcal{G}_{k}$. Due to the lack of space proofs (available in Appendix B) are omitted.

Theorem 3. For every $k \in \omega$ the basis $N_{\Theta_{k}}$ of the $\Theta_{k}$ operation equals the family promise $\left(\mathcal{G}_{k}\right) \stackrel{\text { def }}{=}\left\{X \subseteq \mathbb{A}_{k}: \exists\right.$ has a winning strategy in $\mathcal{G}_{k}$ with promise $\left.X\right\}$.

Corollary 1. For each $k$ and $\left(E_{s}\right)_{s \in \mathbb{A}_{k}}$ we have $\Theta_{k}\left(\left(E_{s}\right)_{s \in \mathbb{A}_{k}}\right)=\mathcal{G}_{k}\left(\left(E_{s}\right)_{s \in \mathbb{A}_{k}}\right)$.

\section{Relation between $\mathcal{R}$-sets and the index hierarchy}

In this section we prove the main result of this work, that is Theorem 1. As a preliminary step, it is convenient to define a variant of game tree languages defined on countable trees. This will simplify the connection with Matryoshka games which are played on countably branching structures. Let $\operatorname{Tr}_{i, k}^{\omega}$ be the space of labelled $\omega$-trees $t: \omega^{*} \rightarrow\{\exists, \forall\} \times\{i, \ldots, k, \top, \perp\}$. Each $t \in \operatorname{Tr}_{i, k}^{\omega}$ is naturally interpreted as a parity game on the countable tree structure, with the possibility of terminating at leaves, labelled by $\top$ and $\perp$, which are winning for $\exists$ and $\forall$, respectively. We also require (1) that in the root there is a vertex $(P, k)$ where $P=\exists$ if $i$ is even and $P=\forall$ if $i$ is odd and (2) that the tree is alternating, that is $\exists$ and $\forall$ make moves in turns.

Definition 7. $\mathcal{W}_{i, k}^{\omega} \subseteq \operatorname{Tr}_{i, k}^{\omega}$ is the set of $\omega$-trees such that $\exists$ has a w.s.

An easy argument (see Section B.7) shows that dropping conditions (1) and (2) gives a Wadge equivalent language. The following routine lemma (see Section B. 8 for a proof) establishes the connection between $\omega$-branching game tree languages $\mathcal{W}_{i, k}^{\omega}$ and binary (as in Section 2) game tree languages $\mathcal{W}_{i, k}$.

Lemma 2. For $i<k$ the language $\mathcal{W}_{i, k}$ is Wadge equivalent to $\mathcal{W}_{i+1, k}^{\omega}$. In particular $\mathcal{W}_{0,1} \sim_{W} \mathcal{W}_{1,1}^{\omega}$ and $\mathcal{W}_{1,3} \sim_{W} \mathcal{W}_{0,1}^{\omega}$.

The fact that $\mathcal{W}_{i, k}$ corresponds to $\mathcal{W}_{i+1, k}^{\omega}$ reflects the cost of the translation of $\omega$-branching games into binary games: an extra priority is required to mimic countably many choices by iterating binary choices. Thanks to this lemma, in Theorem 1 one can replace the languages $\mathcal{W}_{k-1,2 k-1}$ with the languages $\mathcal{W}_{k, 2 k-1}^{\omega}$.

First, we show that every $\mathcal{W}_{k, 2 k-1}^{\omega}$ is indeed an $\mathcal{R}$-set. We will do so by explicitly constructing a family $\mathcal{E}_{k}=\left\{E_{s} \mid s \in \mathbb{A}_{k}\right\}$ of clopen sets in $\operatorname{Tr}_{k, 2 k-1}^{\omega}$ such that $\Theta_{k}\left(\mathcal{E}_{k}\right)=\mathcal{W}_{k, 2 k-1}^{\omega}$, where $\mathbb{A}_{k}$ is the arena of the operation $\Theta_{k}$. The construction requires some effort. First we recall, from Section 3 that the arena of the operation $\bigcup \circ \bigcap$ is $\mathbb{A}_{0}=\{\langle n, m\rangle: n, m \in \omega\}$ (the pairs of natural numbers) and from the definition of the transformation $\mathcal{R}$ we have $\mathbb{A}_{k}=\left(\mathbb{A}_{k-1}\right)^{*}$. Thus, for all $k \in \omega$, $\mathbb{A}_{k}$ is a set of nested sequences of pairs of natural numbers. For a sequence $s \in \mathbb{A}_{k}$ we define the maps flatten and prioritiesMap such that flatten $(s) \in \mathbb{A}_{0}^{\star}$ and prioritiesMap $(s) \in \omega^{*}$. The map flatten takes a nested sequence in $\mathbb{A}_{k}$ and returns the "flattened" sequence, that is all the braces are removed, for example flatten $((((\langle 2,15\rangle)),((\langle 7,5\rangle),(\langle 6,4\rangle))))=(\langle 2,15\rangle,\langle 7,5\rangle,\langle 6,4\rangle)$. The function 
prioritiesMap computes (see Section B.9) the number of the closing brackets after each pair of natural numbers:

$$
\text { prioritiesMap }((((\langle 2,15\rangle)),((\langle 7,5\rangle),(\langle 6,4\rangle))))=(2,1,3) \text {. }
$$

We also define treeMap $(t, s)$ where $t \in \operatorname{Tr}_{k, 2 k-1}^{\omega}$ and $s \in \mathbb{A}_{k}$. Since we limited our attention to alternating trees, each vertex in the $\omega$-branching tree $t$ can be identified with a sequence of pairs of natural numbers. Then, if $s \in \mathbb{A}_{k}$, the function $\operatorname{treeMap}(t, s)$ computes first flatten $(s)$ and returns the sequence of priorities assigned to the vertices along the path of $t$ indicated by flatten $(s)$. On Figure 3 we have an example of a tree $t$ where

$$
\operatorname{treeMap}(t,(((\langle 2,15\rangle)),((\langle 7,5\rangle),(\langle 6,4\rangle))))=(2,1,3) \text {. }
$$

Define $\mathcal{E}_{k}=\left\{E_{s}: s \in \mathbb{A}_{k}\right\}$ such that for $t \in$ $\operatorname{Tr}_{k, 2 k-1}^{\omega}$ we have $t \in E_{s}$ iff for

- $v=\operatorname{prioritiesMap}(s)$,

$-b=\operatorname{treeMap}(t, s)$,

$-L=\min \{k \in \omega: v(k) \neq b(k)\}$

$v \neq b$ holds, and either $b(L)=\top$ or

$$
\min (b(L), v(L)) \equiv 0 \quad(\bmod 2) .
$$

It is simple to verify that the sets $E_{s}$ are indeed clopen in the space $\operatorname{Tr}_{k, 2 k-1}^{\omega}$ (for a definition of the topology see, e.g. [2]).

Theorem 4. $\forall_{k \geq 1} \Theta_{k}\left(\mathcal{E}_{k}\right)=\mathcal{W}_{k, 2 k-1}^{\omega}$.

Proof. The proof is based on Matryoshka games. Consider a tree $t \in \operatorname{Tr}_{k, 2 k-1}^{\omega}$ and assume that player $P \in\{\exists, \forall\}$ has a winning strategy $\sigma$ on the tree $t$. We claim that $P$ has a winning strategy in the Matryoshka game $\mathcal{G}_{k}$ with promise $\mathcal{E}_{k}$. From this fact the theorem will follow by an application of Corollary 1 and Proposition 1. For the

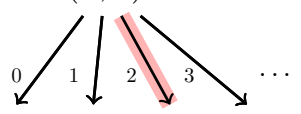

$(\exists, 2)(\exists, 2)(\exists, 2)(\exists, 2) \cdots$


Fig. 3. An illustration of treeMap. simplicity we assume that $P=\exists$, the opposite case is analogous.

We will simulate the game on $t$ in the Matryoshka game $\mathcal{G}_{k}$. A play in $\mathcal{G}_{k}$ consists of playing pairs of numbers (corresponding to moves in $t$ ) in the copies of $\mathcal{G}_{0}$ and additionally of deciding whether to exit an $j$-layer of the game or not. We say that a play in $\mathcal{G}_{k}$ is fair if whenever the players encounter a priority $k+j$ in $t$ then they exit exactly $j$ first layers of $\mathcal{G}_{k}$ (i.e. the layer $j+1$ is reached) and if they encounter a symbol $\perp$ or $\top$ then the players exit all the layers of $\mathcal{G}_{k}$.

Let $\exists$ use the original strategy $\sigma$ in the copies of $\mathcal{G}_{0}$ and play "fairly" as long as $\forall$ does. If $\forall$ also plays "fairly" then the play is winning for $\exists$ : either $T$ is reached 
in $t$ and $\exists$ wins since $t \in E_{s}$ or the play is infinite and $\exists$ wins by the parity condition - the priorities visited in $\mathcal{G}_{k}$ agree with those visited in $t$, see (5).

If $\forall$ does not play "fairly" (i.e. when a priority $k+j$ is reached in $t$ he does not exit the $l$-layer of $\mathcal{G}_{k}$ with $l \leq j$ or he exits the $(j+1)$-layer of $\left.\mathcal{G}_{k}\right)$ then $\exists$ uses the following counter-strategy: whenever possible she exits the current layer of $\mathcal{G}_{k}$. There are two possible developments of such a play. The first case is that $\forall$ allows to exit the whole game and then $\exists$ wins thanks to (6). Now assume that $\forall$ never allows the game to reach a terminal position. In that case, let $j$ be maximal such that the $j$-layer of $\mathcal{G}_{k}$ is visited infinitely often. By (5) we know that the limes superior of the priorities visited in $\mathcal{G}_{k}$ is $k+j-1$ and since $\forall$ is the owner of the vertices in the $j$-layer of $\mathcal{G}_{k}$ so $k+j-1 \equiv 0(\bmod 2)$. Therefore, $\exists$ wins the play by the parity condition.

Theorem 5. Let $L=\Theta_{k}\left(E_{s}\right)$ be a set obtained using the $\Theta_{k}$ operation applied to a family of clopen subsets $\left(E_{s}\right)_{s \in \mathbb{A}_{k}}$ with $E_{s} \subseteq Y$ in a Polish space $Y$. Then, there exists a continuous reduction $f: Y \rightarrow \operatorname{Tr}_{k, 2 k-1}^{\omega}$ such that $f^{-1}\left(\mathcal{W}_{k, 2 k-1}^{\omega}\right)=L$.

Sketch. The operation $\Theta_{k}$ is presented as the corresponding Matryoshka game. This is a parity game played on a countable arena and thus continuously reducible to $\mathcal{W}_{k, 2 k-1}^{\omega}$. A detailed proof is available in Appendix B.

Theorems 4 and 5 imply that the language $\mathcal{W}_{k, 2 k-1}^{\omega}$ is complete for the $k$-th level of the hierarchy of $\mathcal{R}$-sets. Theorem 1 follows from Lemmas 1 and 2 .

\section{Continuity of measures on $\mathcal{W}_{i, k}$}

For an odd $k \in \omega$ the language $\mathcal{W}_{i, k}$ admits a natural decomposition into simpler approximant sets $\mathcal{W}_{i, k}^{\alpha}$, for $\alpha<\omega_{1}$. In the proof of the determinacy of $\mathrm{pL} \mu$ games of [19], the following special continuity property is required: $\sup _{\alpha<\omega_{1}} \mu\left(\mathcal{W}_{i, k}^{\alpha}\right)=$ $\mu\left(\mathcal{W}_{i, k}\right)$. Since the increasing chain $\mathcal{W}_{i, k}^{\alpha}$ is uncountable, the property does not follow from the standard $\sigma$-continuity of measures. As observed in [19], the property holds under Martin Axiom at $\aleph_{1}\left(\mathrm{MA}_{\aleph_{1}}\right)$. The problem of whether the property holds in ZFC alone is left open (see Item 2 of Section 8.2 in [19]). The following theorem gives a partial answer to this problem.

Theorem 6. The continuity property holds in ZFC for $\mathcal{W}_{0,1}$. Let $k$ be an odd number, $i<k$. For $\mathcal{W}_{i, k}$ the continuity property holds assuming the determinacy of Harrington's games ${ }^{7}$ with arbitrary analytic winning sets.

An introduction of the sets $\mathcal{W}_{i, k}^{\alpha}$ and a proof of this theorem is given in Appendix C. Despite the fact that the assumption of determinacy of Harrington's games with analytic winning sets is not a consequence of ZFC, this result is an improvement over the application of $\mathrm{MA}_{\aleph_{1}}$ because $\mathrm{MA}_{\aleph_{1}}$ implies determinacy of Harrington's games, while the converse implication does not hold. Furthermore, our proof based on determinacy, shows that continuity property does not depend on cardinality assumptions such as the Continuum Hypothesis.

\footnotetext{
7 See, e.g., [11, Section 33.5] for details about this type of games.
} 


\section{Conclusion}

The notion of $\mathcal{R}$-sets is a robust concept and admits natural variations. One can equivalently work in arbitrary (not zero-dimensional) Polish spaces and start from a basis of, e.g. Borel sets rather than clopens. The family of operations $\Theta_{k}=(\operatorname{co}-\mathcal{R})^{k}(\bigcup \circ \bigcap)$ can be replaced by, e.g. either $(\operatorname{co}-\mathcal{R})^{k}(\bigcup)$ or $(\operatorname{co}-\mathcal{R})^{k}(\bigcap)$. Similarly, one can consider binary rather than countably branching, Matryoshka games. The notion of $\mathcal{R}$-sets remains unchanged in these alternative setups. The specific choices of this paper have been made in an attempt to find a balance between the modern language of parity games and older literature such as [8].

\section{References}

1. A. Arnold. The $\mu$-calculus alternation-depth hierarchy is strict on binary trees. ITA, 33(4/5):329-340, 1999.

2. A. Arnold and D. Niwiński. Continuous separation of game languages. Fundamenta Informaticae, 81(1-3):19-28, 2007.

3. R. Barua. Studies in Set-Theoretic Hierarchies: From Borel sets to R-sets. PhD thesis, Indian Statistical Institute, Calcutta, 1986.

4. D. Blackwell. Borel-programmable functions. Ann. of Probability, 6:321-324, 1978.

5. J. Bradfield. The modal mu-calculus alternation hierarchy is strict. In $C O N C U R$ '96: Concurrency Theory, volume 1119 of LNCS, pages 233-246. Springer, 1996.

6. J. C. Bradfield. Fixpoints, games and the difference hierarchy. ITA, 37(1):1-15, 2003.

7. J. C. Bradfield, J. Duparc, and S. Quickert. Transfinite extension of the mucalculus. In CSL, pages 384-396, 2005.

8. J. P. Burgess. Classical hierarchies from a modern standpoint. II. R-sets. Fund. Math., 115(2):97-105, 1983.

9. H. V. Daniele Varacca and G. Winskel. Probabilistic event structures and domains. In Proceeding of CONCUR, 2004.

10. A. Facchini, F. Murlak, and M. Skrzypczak. Rabin-mostowski index problem: A step beyond deterministic automata. In LICS, pages 499-508, 2013.

11. T. Jech. Set Thery. Springer Monographs in Mathematics. Springer, 2002.

12. V. G. Kanover. A. N. Kolmogorov's ideas in the theory of operations on sets. Uspekhi Mat. Nauk, 43(6(264)):93-128, 1988.

13. A. Kechris. Classical descriptive set theory. Springer-Verlag, New York, 1995.

14. A. Kolmogorov. Operations sur des ensembles (in Russian, summary in French). Mat. Sb., 35:415-422, 1928.

15. D. Kozen. Results on the propositional mu-calculus. In Theoretical Computer Science, pages 333-354, 1983.

16. M. Kudlek. Probability in Petri Nets. Fundamenta Informaticae, 67(1), 2005.

17. A. A. Lyapunov. $\mathcal{R}$-sets. Trudy Mat. Inst. Steklov., 40:3-67, 1953.

18. H. Michalewski and D. Niwiński. On topological completeness of regular tree languagess. Logic and Program Semantics, pages 165-179, 2012.

19. M. Mio. Game Semantics for Probabilistic $\mu$-Calculi. PhD thesis, School of Informatics, University of Edinburgh, 2012.

20. M. Mio. Probabilistic Modal $\mu$-Calculus with Independent product. Logical Methods in Computer Science, 8(4), 2012.

21. E. Selivanowski. Sur une classe d'ensembles effectifs (ensembles C) (in Russian, summary in French). Mat. Sb., 35:379-413, 1928.

22. G. Winskel. Distributed probabilistic strategies. In Proceedings of MFPS, 2013. 


\section{A Acknowledgments}

The third author would like to thank Alex Simpson for innumerable suggestions and fruitful collaborations. The second and the third authors are supported by the Expressiveness of Modal Fixpoint Logics project realized within the 5/2012 Homing Plus programme of the Foundation for Polish Science, co-financed by the European Union from the Regional Development Fund within the Operational Programme Innovative Economy ("Grants for Innovation").

\section{B Remaining proofs}

\section{B.1 Proofs of basic facts about $\mathcal{R}$-sets}

Lemma 1. The $k$-th level of $\mathcal{R}$-sets is closed under pre-images of continuous functions.

Proof. Because pre-image of a clopen set is also clopen, the following equation finishes the proof

$$
\begin{aligned}
& f^{-1}\left(\Theta\left(\left\{E_{s}\right\}_{s}\right)\right)=f^{-1}\left(\bigcup_{N \in N_{\Theta}} \bigcap_{s \in N} E_{s}\right)= \\
& =\bigcup_{N \in N_{\Theta}} \bigcap_{s \in N} f^{-1}\left(E_{s}\right)= \\
& =\Theta\left(\left\{f^{-1}\left(E_{s}\right)\right\}_{s}\right) \text {. }
\end{aligned}
$$

Lemma 3. For a given operator $\Theta$ the classes of sets which can be obtained by operators $\mathcal{R} \Theta$ and $\mathcal{R} \Theta \circ \mathcal{R} \Theta$ are the same.

For a proof of this lemma we refer to [13, Proposition 25.6] which is stated for the Souslin operator; the same proof works when we replace $\mathcal{A}$ by $\mathcal{R} \Theta$.

Lemma 4. Let $k>0$ and assume that $f: X \rightarrow Y$ is a function between two Polish spaces $X, Y$ such that for every clopen set $C \subset Y$ the preimage $f^{-1}[C]$ is on the $k$-th level of the $\mathcal{R}$-hierarchy. Then for every $\mathcal{R}$-set $A \subset Y$ on the $k$-th level of the $\mathcal{R}$-hierarchy the preimage $f^{-1}[A]$ also belongs to the $k$-the level of the $\mathcal{R}$-hierarchy.

Proof. First observe that similarly as in (7) we have

$$
f^{-1}\left(\mathcal{R} \Theta_{k}\left(\left\{E_{s}\right\}_{s}\right)\right)=\mathcal{R} \Theta_{k}\left(\left\{f^{-1}\left(E_{s}\right)\right\}_{s}\right) .
$$

Now, by the assumption, each of the sets $f^{-1}\left(E_{s}\right)$ is of the form

$$
\mathcal{R} \Theta\left(\left(F_{s, s^{\prime}}\right)_{s^{\prime} \in \mathbb{A}_{\mathcal{R} \Theta}}\right) \text {. }
$$

Therefore,

$$
f^{-1}\left(\mathcal{R} \Theta\left(\left\{E_{s}\right\}_{s}\right)\right)=\mathcal{R} \Theta \circ \mathcal{R} \Theta\left(F_{s, s^{\prime}}\right)
$$

and the thesis holds by normality of the operator $\mathcal{R} \Theta$. 


\section{B.2 Proof of the preservation Theorem 2}

Theorem 2. If $\Gamma$ and $\Theta$ preserve measurability then $\Gamma \circ \Theta, \mathcal{R} \Gamma$, and co- $\Gamma$ preserve measurability.

Preservation of measurability under $\mathcal{R}$ transformation follows immediately from Lemma 6. More elementary preservation of measurability under two other transformation co- and composition is discussed in Lemma 8. In the proof of Lemma 6 we will need the following

Lemma 5 ([13, Theorem 29.12]). For every set $B \subseteq \mathbb{R}$ there exists a measurable set $\bar{B} \supset B$ such that for every measurable set $C, B \subseteq C \subseteq \bar{B}$ one has $\mu(\bar{B} \backslash C)=0$.

Lemma 6 ([17, Theorem 4]). If $\Theta$ preserves measurability then $\mathcal{R} \Theta$ preserve measurability.

The proof below was proposed by Barua in [3]; the method is an adaptation of a classical proof of Marczewski regarding the Souslin $\mathcal{A}$ operation.

Proof. Let $\mathbb{A}$ be the arena of $\Theta,\left\{E_{s}\right\}_{s \in \mathbb{A}<\omega}$ be a family of clopen sets. Let $E^{s}$ denote the partial result of $\mathcal{R} \Theta$ application, i.e. $E^{s}=\mathcal{R} \Theta\left(\left\{E_{s t} \mid t \in \mathbb{A}^{<\omega}\right\}\right)$. By definition $E^{\epsilon}=\mathcal{R} \Theta\left(\left\{E_{s}\right\}_{s \in \mathbb{A}<\omega}\right)$. By Lemma 5 one can find measurable sets $\bar{E}^{s} \supseteq E^{s}$. Without loss of generality one can assume, that $\bar{E}^{s} \subseteq E_{s}$. In order to complete the proof of preservation of measurability it will be enough to show the following

\section{Lemma 7.}

$$
\bar{E}^{\epsilon} \backslash E^{\epsilon} \subseteq \bigcup_{s}\left(\bar{E}^{s} \backslash \Theta\left(\left\{\bar{E}^{s t} \mid t \in \mathbb{A}\right\}\right)\right)
$$

Proof. Suppose not. Then there is an $x \in \bar{E}^{\epsilon} \backslash E^{\epsilon}$ such that $\forall s \quad x \in \bar{E}^{s} \rightarrow x \in$ $\Theta\left(\left\{\bar{E}^{s t} \mid t \in \mathbb{A}\right\}\right)$. Since

$$
x \in \Theta\left(\left\{\bar{E}^{s t} \mid t \in \mathbb{A}\right\}\right) \Leftrightarrow \exists \eta \in N \forall n \in \eta\left(x \in \bar{E}^{s n}\right)
$$

by induction on length sequence $s$ we get

$$
\exists \eta_{0} \in N \forall n_{0} \in \eta_{0} \quad \exists \eta_{1} \in N \forall n_{1} \in \eta_{1} \ldots \quad \forall k\left(x \in \bar{E}^{\left(n_{0}, \ldots, n_{k}\right)}\right) .
$$

But $\bar{E}^{s} \subseteq E_{s}$, so the statement above shows that $x \in E^{\epsilon}$, a contradiction.

In Lemma 7 the set on the right hand side has measure 0 , so every its subset has external measure 0 . Hence, it is measurable. Set $\bar{E}^{\epsilon}$ is measurable by definition, so $E^{\epsilon}$ also is measurable.

This finished the proof of the preservation of measurability under $\mathcal{R}$ transformation. It remains to prove the following elementary 
Lemma 8. If $\Gamma$ and $\Theta$ preserves measurability then $\Gamma \circ \Theta$ and co- $(\Gamma)$ preserve measurability.

Proof. By definition application $\Gamma \circ \Theta$ to clopen sets is an application of $\Gamma$ to results of application $\Theta$ to clopen sets. Since $\Theta$ preserves measurability, $\Gamma$ is applied to measurable sets. Since $\Gamma$ is also preservers measurability, at the end we get measurable set.

Assuming that $\Gamma$ preserves measurability, co- $(\Gamma)$ also preserves measurability thanks to the following equality

$$
\operatorname{co-} \Theta\left(\left\{E_{s}\right\}_{s \in \mathbb{A}}\right)=\Theta\left(\left\{E_{s}^{c}\right\}_{s \in \mathbb{A}}\right)^{c}
$$

This Lemma implicitly appears in [17] and is considered a part of mathematical folklore.

\section{B.3 Examples showing relation between transformation $\mathcal{R}$ and operations $\bigcup, \bigcap$ and $\mathcal{A}$}

First we notice that $N_{U}=\{\{n\} \mid n \in \mathbb{N}\}$ and $N_{\bigcap}=\{\mathbb{N}\}$

Example 1. We claim, that $\mathcal{R} \bigcup=\mathcal{A}$, the Souslin operation, and the basis for $\mathcal{R} \bigcup$ is equal to

$$
N_{\mathcal{R} \cup}=\left\{\{v \mid v \text { is a finite prefix of } s\}: s \in \omega^{\omega}\right\}
$$

Consider arbitrary $T \in N_{\mathcal{R}} \cup$. From the definition of the operation $\mathcal{R} \bigcup, T$ is a tree such that for every node $v \in T$ the set $\{n: v n \in T\}$ is a member of $N_{U}$. Hence $T$ is an infinite path. Take any family of clopen sets $E_{s}\left(s \in \omega^{*}\right)$. Then

$$
\mathcal{R} \bigcup\left(E_{s}\right)=\sum_{T \in N_{\mathcal{R} \cup}} \bigcap_{s \in T} E_{s}
$$

which is exactly the definition of the Souslin operation.

Example 2. From the definition of co- follow equalities

$$
\text { co- } U=\bigcap \text { and co- } \bigcap=\bigcup \text {. }
$$

Indeed. $N_{\text {co- } U}$ consists of sets which have nonempty intersection with every singleton. But there is only one such set: $\mathbb{N}$. Hence, $N_{\text {co- } U}=N_{U}$.

The second equality is a bit more involved. By pipelining definitions we have

$$
x \in \operatorname{co}-\bigcap\left(\left\{E_{s}\right\}_{s \in \mathbb{N}}\right) \Leftrightarrow \exists S \subset \mathbb{N}\left(S \cap \mathbb{N} \neq \emptyset \wedge \forall s \in S x \in E_{s}\right) .
$$

The smaller set $S$ is the easier the right condition is. Therefore the right condition is equivalent to $\exists s \in \mathbb{N} x \in E_{s}$. In other words $x \in \bigcup\left(\left\{E_{s}\right\}_{s \in \mathbb{N}}\right)$. 


\section{B.4 Proof of determinacy for Matryoshka games (Proposition 1)}

Proposition 1. If $\mathcal{G}$ is a Matryoshka game with play labels $\mathbb{A}^{\mathcal{G}}$ and $X \subseteq \mathbb{A}^{\mathcal{G}}$ then exactly one of the players has a winning strategy in $\mathcal{G}$ with promise $X$.

Proof. By reduction to the standard parity games: first, we can assume that we play on the unravelling of the arena with additional loop-edges on elements of $F^{\mathcal{G}}$. For a given promise $X \subseteq \mathbb{A}^{\mathcal{G}}$ we can set the priorities on $F^{\mathcal{G}}$ such that the position in the unravelling corresponding to $f \in F^{\mathcal{G}}$ is winning for $\exists$ if and only if the label of the unique play reaching this position belongs to $X$.

A winning strategy for $P$ in the obtained game gives a $X$-winning strategy for $P$ in the original game.

\section{B.5 Formal introduction of games $\mathcal{G}_{k}$}

Recall that a Matryoshka game is a tuple

$$
\mathcal{G}=\left\{V^{\mathcal{G}}=V_{\exists}^{\mathcal{G}} \sqcup V_{\forall}^{\mathcal{G}}, F^{\mathcal{G}}, E^{\mathcal{G}}, v_{I}^{\mathcal{G}}, \Omega^{\mathcal{G}}, \mathbb{A}^{\mathcal{G}}, \text { label }^{\mathcal{G}}\right\} .
$$

Let us define precisely all the elements:

$-V^{\mathcal{G}}$ is a countable set of positions of the game,

$-F^{\mathcal{G}}$ is a countable set of terminal positions of the game,

$-E^{\mathcal{G}} \subseteq V^{\mathcal{G}} \times\left(V^{\mathcal{G}} \cup F^{\mathcal{G}}\right)$ is the edge relation,

$-v_{I}^{\mathcal{G}} \in V^{\mathcal{G}}$ is the initial position,

- $\Omega^{\mathcal{G}}: V^{\mathcal{G}} \rightarrow\{i, \ldots, k\} \subseteq \omega$ is the priority function,

$-\mathbb{A}^{\mathcal{G}}$ is a set of play labels,

- label ${ }^{\mathcal{G}}:\left(V^{\mathcal{G}}\right)^{*} F^{\mathcal{G}} \rightarrow \mathbb{A}^{\mathcal{G}}$ is a function assigning to finite plays their play labels.

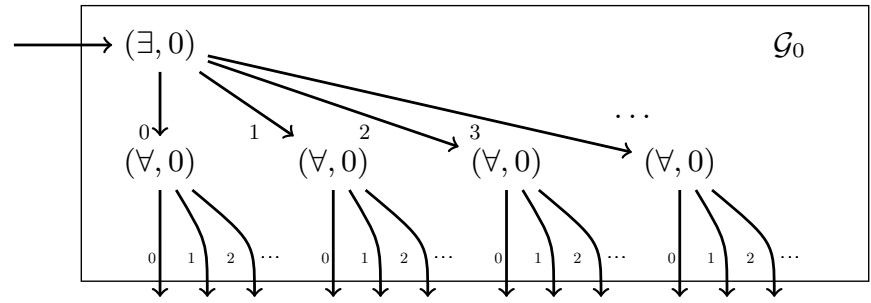

Fig. 4. Repeated Figure 1.

Game $\mathcal{G}_{0}$. Let the arena $V^{\mathcal{G}_{0}}$ consist of $V_{\exists}^{\mathcal{G}_{0}}=\left\{e_{0}\right\}$ and $V_{\forall}^{\mathcal{G}_{0}}=\left\{a_{0}, a_{1}, \ldots\right\}$ and let $F^{\mathcal{G}_{0}}=\left\{f_{n, m}: n, m \in \mathbb{N}\right\}$. Let $E^{\mathcal{G}_{0}}$ contain pairs of the form $\left(e_{0}, a_{n}\right)$ and $\left(a_{n}, f_{n, m}\right)$ for $n, m \in \mathbb{N}$. Let $\mathbb{A}^{\mathcal{G}_{0}}=\omega^{2}$. Note that all the plays of $\mathcal{G}_{0}$ are finite and of the form $\pi=\left(e_{0}, a_{n}, f_{n, m}\right)$. For such a play, let label ${ }^{\mathcal{G}_{0}}(\pi)=(n, m)$. Let $\Omega^{\mathcal{G}_{0}}: V^{\mathcal{G}_{0}} \rightarrow\{0\}$ be the constant function. 


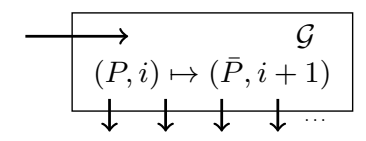

Fig. 5. The game co- $\mathcal{G}$.

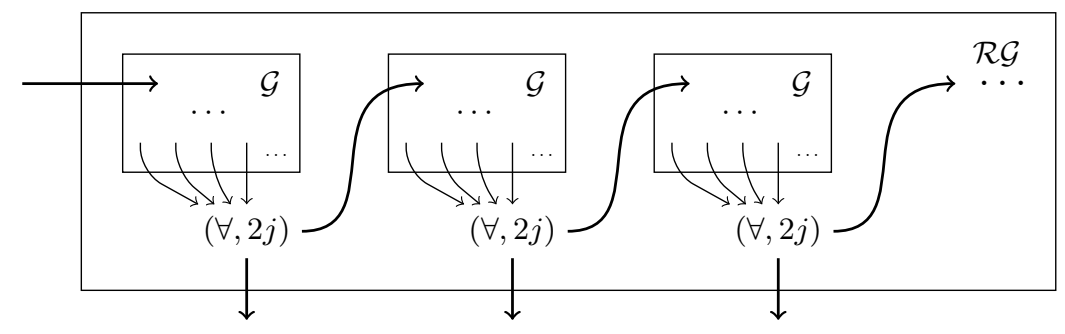

Fig. 6. Repeated Figure 2.

Game co- $\mathcal{G}$. As defined in Section 4 , the game co- $\mathcal{G}$ is obtained from $\mathcal{G}$ by replacing the players and increasing all the priorities by 1 .

Game $\mathcal{R G}$. Formally, the set of positions $V^{\mathcal{R G}}$ of $\mathcal{R G}$ is defined as $\left\{a_{0}, a_{1}, \ldots\right\} \sqcup$ $\omega \times V^{\mathcal{G}}$. Each vertex $a_{n}$ belongs to $\forall$ (i.e. $a_{n} \in V_{\forall}^{\mathcal{R G}}$ ). A vertex $(n, v) \in \omega \times V^{\mathcal{G}}$ belongs to a player $P$ if and only if $v \in V_{P}^{\mathcal{G}}$. $\mathcal{R} \mathcal{G}$ has infinitely many terminal positions $f_{0}, f_{1}, \ldots$ The priority function on $\omega \times V^{\mathcal{G}}$ is the same as in $\mathcal{G}$. All the vertices $a_{n}$ have priority $2 j$. The edges in $\mathcal{R G}$ are of the following forms:

- if $\left(v, v^{\prime}\right) \in E^{\mathcal{G}}$ with $v, v^{\prime} \in V^{\mathcal{G}}$ then $\left((n, v),\left(n, v^{\prime}\right)\right) \in E^{\mathcal{R G}}$ for $n \in \mathbb{N}$,

- if $(v, f) \in E^{\mathcal{G}}$ with $v \in V^{\mathcal{G}}$ and $f \in F^{\mathcal{G}}$ then $\left((n, v), a_{n}\right) \in E^{\mathcal{R G}}$ - instead of a terminal position of $\mathcal{G}$ we move to the successive vertex of $\forall$,

- additionally, we add edges $\left(a_{n},\left(n+1, v_{I}^{\mathcal{G}}\right)\right) \in E^{\mathcal{R G}}$ and $\left(a_{n}, f_{n}\right) \in E^{\mathcal{R G}}$.

Let the initial position of $\mathcal{R G}$ be $\left(0, v_{I}^{\mathcal{G}}\right)$.

The crucial part of the definition of the transformation $\mathcal{R}$ are the labels, see (4). Consider a finite play $\pi$ that reaches a terminal position $f_{n}$ of $\mathcal{R G}$. Such a play has lasted for $n$ rounds until it reached the terminal position $f_{n}$. In that case, the play $\pi$ is of the form:

$$
a_{0} \pi_{0} a_{1} \pi_{1} \ldots \pi_{n-1} a_{n} f_{n}
$$

where $\pi_{i}$ corresponds to a play in $\mathcal{G}$. Let $x_{i}$ be the label assigned by $\mathcal{G}$ to the play $\pi_{i}$ and let

$$
\operatorname{label}^{\mathcal{R G}}(\pi)=\left(x_{0}, x_{1}, \ldots, x_{n-1}\right)
$$

\section{B.6 Proof of Theorem 3}

Theorem 3. For every $k \in \omega$ the basis $N_{\Theta_{k}}$ of the $\Theta_{k}$ operation equals the family $\operatorname{promise}\left(\mathcal{G}_{k}\right) \stackrel{\text { def }}{=}\left\{X \subseteq \mathbb{A}_{k}: \exists\right.$ has a winning strategy in $\mathcal{G}_{k}$ with promise $\left.X\right\}$. 
Proof. The proof goes by induction. First take $k=0$. Note that the following family forms a basis of $\Theta_{0}=\bigcup \circ \bigcap$ :

$$
N_{\Theta_{0}}=\left\{N \subseteq \omega^{2}: \exists_{n} \forall_{m}(n, m) \in N\right\} .
$$

Observe that the strategies of $\exists$ in $\mathcal{G}_{0}$ boil down to selecting the first number $n$. Then $\forall$ selects the second number $m$ and the play ends in a terminal position with label $(n, m)$. Therefore, the family of promises of winning strategies of $\exists$ in $\mathcal{G}_{0}$ coincides with $N_{\Theta_{0}}$.

Now assume that $N_{\Theta}=\operatorname{promise}(\mathcal{G})$, we prove that $N_{\text {co- } \Theta}=$ promise $($ co- $\mathcal{G})$. Let $\mathbb{A}$ be the play labels in $\mathcal{G}$. Observe that the following conditions are equivalent:

$$
\begin{gathered}
X \in N_{\text {co- } \Theta} \text { by the definition of co- } \Theta \\
\forall_{X^{\prime} \in N_{\Theta}} X \cap X^{\prime} \neq \emptyset \\
\mathbb{A} \backslash X \notin N_{\Theta} \text { since } N_{\Theta} \text { is upward-closed }
\end{gathered}
$$

by the inductive assumption

$\exists$ does not have a $(\mathbb{A} \backslash X)$-winning strategy in $\mathcal{G}$

by the definition of co- $\mathcal{G}$

$\forall$ does not have a $X$-winning strategy in co- $\mathcal{G}$

by determinacy (Proposition 1

$\exists$ has a $X$-winning strategy in co- $\mathcal{G}$

by the definition of promise (co- $\mathcal{G})$

$$
X \in \operatorname{promise}(\text { co- } \mathcal{G})
$$

Now assume that $N_{\Theta}=\operatorname{promise}(\mathcal{G})$, we prove that $N_{\mathcal{R} \Theta}=\operatorname{promise}(\mathcal{R G})$. This will finish the inductive proof of the proposition. As above, let $\mathbb{A}$ equal arena $(\mathcal{G})$. Additionally, let $\mathcal{G}^{i}$ denote the sub-game of $\mathcal{R G}$ corresponding to the $i$-th copy of $\mathcal{G}$ (formally, $\mathcal{G}^{i}$ contains vertices of the form $(i, v)$ ).

First assume that $\sigma$ is a $X$-winning strategy for $\exists$ in $\mathcal{R G}$. We need to show that $X \in N_{\mathcal{R G}}$. Clearly $\epsilon \in X$ since $\forall$ can move directly from $a_{0}$ to $e_{0}$. Let $\bar{s} \in X$. We need to show that $\{x: \bar{s} x \in X\}$ is an element of $N_{\mathcal{G}}$. Let $i=|\bar{s}|$ be the length of $s$. Observe that $\bar{s} \in X$ means that there exists a finite play $\pi$ that is consistent with $\sigma$ that goes through the sub-games $\mathcal{G}^{0}, \ldots, \mathcal{G}^{i-1}$ and then to $a_{i}$ and $e_{i}$, formally

$$
\pi=a_{0} \pi_{0} a_{1} \pi_{1} \cdots a_{i-1} \pi_{i-1} a_{i} e_{i} .
$$

Consider the strategy of $\exists \sigma^{\prime}$ in $\mathcal{G}$ obtained as restricting $\sigma$ to sequences that extend $a_{0} \pi_{1} \cdots \pi_{i-1} a_{i}\left(i, v_{I}^{\mathcal{G}}\right)$, where $\left(i, v_{I}^{\mathcal{G}}\right)$ is the initial position in the $i$-copy of $\mathcal{G}$. This strategy is winning with some minimal guarantee $X^{\prime} \subseteq \mathbb{A}$. Note that if there is a play $\pi^{\prime}$ consistent with $\sigma^{\prime}$ such that label ${ }^{\mathcal{G}}\left(\pi^{\prime}\right)=x$ then $\bar{s} x \in X-$ 
directly after the play $\pi^{\prime} \forall$ can decide to move from $a_{i+1}$ to $e_{i+1}$. Therefore, $\sigma^{\prime}$ witnesses that $\{x: \bar{s} x \in X\} \in N_{\mathcal{G}}$.

Now assume that $X \in N_{\mathcal{R} \Theta}$. In particular $X \subseteq \mathbb{A}^{*}$ and for every element $\bar{s} \in X$ we have $\{x: \bar{s} x \in X\} \in N_{\mathcal{G}}$. We need to construct a $X$-winning strategy $\sigma$ of $\exists$ in $\mathcal{R G}$. The strategy is defined inductively, between successive sub-games $\mathcal{G}^{i}$. The invariant says, that if a play $\pi$ consistent with $\sigma$ reaches the node $e_{i}$ then label ${ }^{\mathcal{R}}(\pi) \in X$. Assume that we have reached $a_{i}$ after a play $\pi$ such that the label of $\pi e_{i}$ is $\bar{s}$. By the invariant, we know that $\bar{s} \in X$. In particular, there exists a winning strategy $\sigma^{\prime}$ of $\exists$ in $\mathcal{G}$ with the guarantee $\{x: \bar{s} x \in X\}$. Let $\sigma$ follow the decisions of $\sigma^{\prime}$ until reaching a terminal position of $\mathcal{G}$ (i.e. the position $a_{i+1}$ in $\mathcal{R G}$ ). We now prove that $\sigma$ is $X$-winning. Let $\pi$ be a play consistent with $\sigma$. There are the following cases:

$-\pi$ is a finite play and by the above invariant label ${ }^{\mathcal{R G}}(\pi) \in X$.

$-\pi$ is an infinite play that stays from some point on in one of the sub-games $\mathcal{G}^{i}$. In that case $\pi$ is winning for $\exists$ since it contains a winning play in $\mathcal{G}$ as a suffix.

$-\pi$ is an infinite play that passes through infinitely many sub-games $\mathcal{G}^{i}$. In that case all the vertices $a_{i}$ are on $\pi$ so

$$
\limsup _{n \rightarrow \infty} \Omega(\pi(n))=2 n
$$

and therefore $\pi$ satisfies the parity condition.

\section{B.7 Elaboration on two versions of $W_{i, k}^{\omega}$ languages and proof of their Wadge equivalence}

First let us recall Definition 7 in the two variants.

Definition 8. As in the case of $\mathcal{W}_{i, k}$ let $\mathcal{W}_{i, k}^{\omega}$ be the trees in $\operatorname{Tr}_{i, k}^{\omega}$ such that $\exists$ has a winning strategy and the following simple technical conditions are satisfied:

1. in the root there is a vertex $(P, k)$ where $P=\exists$ if $i=0$ and $P=\forall$ if $i=1$,

2. for a vertex $v \in \omega^{2 n}$ on an even depth in the tree, if the label of $v$ is of the form $(P, j)$ then the labels of all the vertices $v l \in \omega^{*}$ for $l \in \omega$ are $(\bar{P}, j)$, see the picture below. In other terms, the players appear alternately and the priorities are duplicated every second level.

The $\omega$-branching game tree language is defined as the set $\mathcal{W}_{i, k}^{\omega} \subseteq \operatorname{Tr}_{i, k}^{\omega}$ of trees which are winning for $\exists$.

Lemma 9. Dropping conditions 1. and 2. in Definition 8 defines a language which is Wadge equivalent to $\mathcal{W}_{i, k}^{\omega}$.

Proof. Let us start from an observation, that adding technical requirements regarding the same ranks on two subsequent levels $2 n$ and $2 n+1$ of a given tree and requiring that player $\exists$ and $\forall$ move one after another in turns does not limit generality from the point of view of Wadge reducibility. Namely, as illustrated by Figure 7 we may modify arbitrary graph to fulfil the additional requirements. 


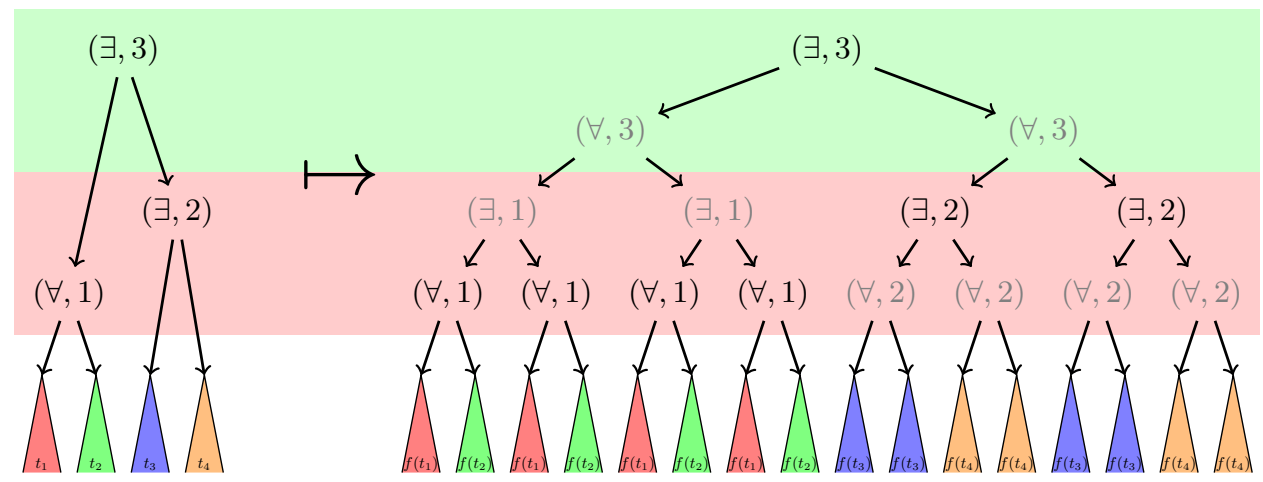

Fig. 7. "Normalization" of game languages - the same technique works for both binary and $\omega$-branching trees.

\section{B.8 Proof of Lemma 2}

Lemma 2. For $i<k$ the language $\mathcal{W}_{i, k}$ is Wadge equivalent to $\mathcal{W}_{i+1, k}^{\omega}$. In particular $\mathcal{W}_{0,1} \sim_{W} \mathcal{W}_{1,1}^{\omega}$ and $\mathcal{W}_{1,3} \sim_{W} \mathcal{W}_{0,1}^{\omega}$.

Proof. Let start with a reduction of $\mathcal{W}_{i+1, k}^{\omega}$ to $\mathcal{W}_{i, k}$. To encode infinite branching we use a standard trick - each leftmost branch $B$ in the binary tree is treated as a one vertex $V$. Right children of vertices in $B$ are treated as children of $V$. To guarantee that a player $P$ who can choose a child of $V$ will always exit branch $B$, we label vertices along $B$ with the lowest possible priority loosing for $P$ (i.e. $i$ or $i+1)$. One should notice, that such labelling does not increase lim sup of a play.

The Wadge reduction of the language $\mathcal{W}_{1,3}^{\omega}$ to $\mathcal{W}_{0,3}$ is shown in Figure 8 below.

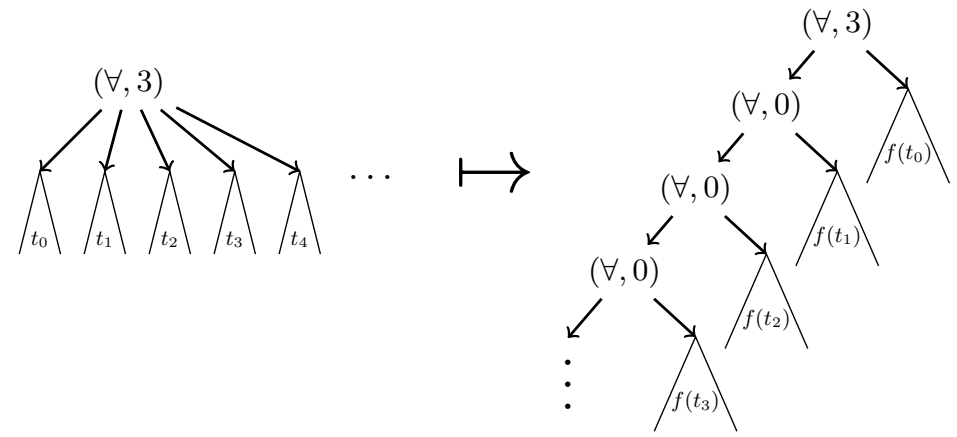

Fig. 8. Reduction of $\mathcal{W}_{1,3}^{\omega}$ to $\mathcal{W}_{0,3}$. 
Technically more involved is a reduction of $\mathcal{W}_{i, k}$ to $\mathcal{W}_{i+1, k}^{\omega}$. The proof below is an adaptation of the proof of Lemmas IV.5 and IV.6 in [10]. Without loss of generality let $i=0$ (i.e. priority winning for $\exists$ ). A continuous reduction $\phi$ maps a tree $t \in \operatorname{Tr}_{i, k}$ into $\phi(t) \in \operatorname{Tr}_{i+1, k}^{\omega}$ and is defined as follows. When we encounter a vertex with priority greater than 0 it is copied without any change - we can duplicate both of the children of this vertex infinitely many times, to make sure that the obtained tree is $\omega$-branching.

The situation is different when we encounter a vertex $v$ with priority 0 . In this case vertex $v^{\prime}=(\forall, 1)$ with $\omega$ children is produced. Intuitively, since priority 0 is loosing for $\forall$ he wants to visit vertices with higher priorities. Let $\tau_{n}$ $(n \in \omega)$ be a list of all strategies of $\forall$ starting from $v$. The successive children of $v^{\prime}$ correspond to the strategies $\left(\tau_{n}\right)_{n \in \mathbb{N}}$. In order to decide children of $\tau_{n}$, we consider possible choices of $\exists$ against strategy $\tau_{n}$. This gives finitely many options which we verbatim copy as children of $\tau_{n}$, unless a priority of such child is 0 . Then we decide that $\forall$ looses and mark it as $T$. We will prove that $\phi$ is a Wadge reduction by showing the following equivalence

$$
t \in \mathcal{W}_{i, k} \quad \text { if and only if } \quad \phi(t) \in \mathcal{W}_{i+1, k}^{\omega} .
$$

The proof is based on the heuristic that if $\forall$ cannot reach a priority greater than 0 then he looses.

Assume first that $\sigma$ is a winning strategy of $\exists$ on the tree $t$. We need to show that $\exists$ wins on $\phi(t)$. We play according to $\sigma$ on $\phi(t)$ until there appears a vertex with priority 0 . Assume now that $\forall$ selected a strategy $\tau_{n}$. Since $\sigma$ and $\tau_{n}$ define a unique answer of $\exists$ in $t$ we can select the counterpart of this answer in the tree $\phi(t)$. Since $\sigma$ is winning, the above strategy either reaches $T$ or the parity condition is satisfied.

Assume now that $\sigma$ is a winning strategy of $\exists$ on $\phi(t)$ and towards a contradiction assume that $\tau$ is a winning strategy of $\forall$ on $t$. We play these two strategies against each other as far as the priority 0 is not reached in $t$. If 0 is reached, then against $\sigma$ we play a finite approximation $\tau_{n}$ of $\tau$ which avoids vertices of rank 0 (if every approximation of $\tau$ contains a 0-labelled leaf then according to the König's lemma we would be able to construct a path in $\tau$ containing only 0-labelled vertices). If $\sigma$ selects a leaf $w$ of $\tau_{n}$ in $\phi(t)$, we mimic the same gameplay in $t$. As a result the visited priorities in $t$ and $\phi(t)$ must be the same, but this contradicts our assumption that the strategies $\sigma$ and $\tau$ are winning for $\exists$ and $\forall$ respectively.

\section{B.9 Precise definition of flatten and prioritiesMap}

We define flatten and prioritiesMap using a natural Haskell data structure. This is a NestedList, an abstraction of a list which naturally allows to consider $\mathbb{A}_{k}$, that is sequences of sequences of... of sequences.

\footnotetext{
-- run at http://www.fpcomplete.com/user/henryk/kolmogorovflatmaps

data NestedList a $=$ Elem a $\mid$ List [NestedList a]

deriving (Show)
} 


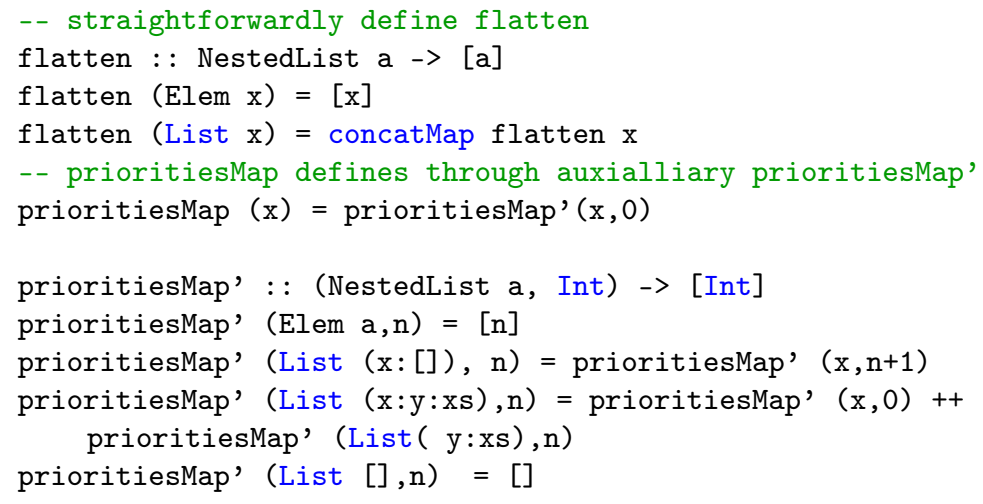

Code can be run locally on a computer or on-line on fpcomplete server; the service allows on-line modifications, in particular playing with more examples at the webpage

http://www.fpcomplete.com/user/henryk/kolmogorovflatmaps

\section{B.10 Proof of Lemma 5}

Lemma 5. Let $L=\Theta_{k}\left(E_{s}\right)$ be a set obtained using the $\Theta_{k}$ operation applied to a family of clopen subsets $\left(E_{s}\right)_{s \in \mathbb{A}_{k}}$ with $E_{s} \subseteq Y$ in a Polish space $Y$. Then, there exists a continuous reduction $f: Y \rightarrow \operatorname{Tr}_{k, 2 k-1}^{\omega}$ such that $f^{-1}\left(\mathcal{W}_{k, 2 k-1}^{\omega}\right)=L$.

Proof. Let $t$ be the unravelling of the arena $V \cup F$ of the game $\mathcal{G}_{k}$ : nodes of $t$ correspond to finite paths in the graph $(V \cup F, E)$. We can assume that $t \subseteq \omega^{*}$ since the arena $V$ is countable. Let us additionally label $t$ : if $\pi$ is a finite path in $\mathcal{G}_{k}$ that ends in a node $v \in V_{P}$ such that $\Omega(v)=j$ then let $t(\pi)=(P, j)$. If $\pi$ ends in a terminal position of $\mathcal{G}_{k}$ then let $t(\pi)=\square$.

By the definition, $t$ is a partial function $t: \omega^{*} \rightarrow\{\exists, \forall\} \times\{k, \ldots, 2 k-1\} \cup\{\square\}$. Additionally observe that the nodes of $t$ that are labelled by $\square$ are leafs of $t$. For nodes $v \in V$ that are not $\omega$-branching in $\mathcal{G}_{k}$, we can duplicate the $E$-successors of $v$ infinitely many times to ensure that each non- $\square$ vertex of $t$ has all the children.

Let us define a function $f$ that assigns to an element $x \in Y$ the tree $t^{\prime}$ obtained from $t$ by replacing each node $\pi$ labelled by $\square$ by the full $\omega$-tree labelled everywhere by:

- $(\exists, \top)$ if $x \in E_{\text {label }} \mathcal{G}_{k}(\pi)$,

- $(\exists, \perp)$ otherwise.

Note that $t^{\prime} \in \operatorname{Tr}_{k, 2 k-1}^{\omega}$ and since the sets $E_{s}$ are clopen so the function $f$ is continuous. Additionally observe that since $t^{\prime}$ is obtained by unravelling of $\mathcal{G}_{k}$ so a strategy in one game can be interpreted as a strategy in the other one and vice versa. 
Observe that $x \in \mathcal{G}_{k}\left(E_{s}\right)$ if and only if $\exists$ has a winning strategy in $\mathcal{G}_{k}$ with the promise $\left\{s: x \in E_{s}\right\}$ if and only if $t^{\prime} \in \mathcal{W}_{k, 2 k-1}^{\omega}$. Therefore, $f$ is a continuous reduction as in the statement. 


\section{Continuity of measures on $\mathcal{W}_{i, k}$}

In this section we study the relation between the measure of a set $\mathcal{W}_{i, k}$ and the measures of it's approximations $\mathcal{W}_{i, k}^{\alpha}$, see Theorem 6 . We start with a formal introduction of the notions used in the statement.

\section{C.1 Ranks}

First we formally introduce the approximations $\mathcal{W}_{i, k}^{\alpha}$. Let us introduce the following definitions, following in [13, Section 2.E]. An $\omega$-forest is a prefix-closed subset of $\omega^{+}$. The set of all $\omega$-forests is denoted $\operatorname{Tr}(\omega)$. An $\omega$-forest is wellfounded if it has no infinite branch. The set of all well-founded $\omega$-forests is denoted $\mathrm{WF} \subseteq \operatorname{Tr}(\omega)$. With every well-founded $\omega$-forest $\tau$ one can bind an ordinal $\operatorname{rank}_{\mathrm{WF}}(\tau)$ called the rank of $\tau$. The definition of $\operatorname{rank}_{\mathrm{WF}}(\tau)$ is inductive: rank $_{\mathrm{WF}}(\emptyset)=0$ and if $\tau$ is non-empty and $\tau_{0}, \tau_{1}, \ldots$ is the list of subforests of $\tau$ rooted at successive roots of $\tau$ then

$$
\operatorname{rank}_{\mathrm{WF}}(\tau)=\sup _{n \in \mathbb{N}} \operatorname{rank}_{\mathrm{WF}}\left(\tau_{n}\right)+1 .
$$

Since the set of nodes of an $\omega$-forest is countable, so $\operatorname{rank}_{W F}(\tau)$ is smaller than $\omega_{1}$ - the first uncountable ordinal. For an $\omega$-forest $\tau$ that is not well-founded we can define $\operatorname{rank}(\tau)$ as $\infty$ - an additional value greater than all ordinals.

Let $i \leq j \leq k$ for an odd $j, t \in \mathcal{W}_{i, k}$, and $\rho \subseteq \operatorname{dom}(t)$ be a winning strategy for $\exists$ on $t$. Consider the set of nodes $V_{j} \subseteq \operatorname{dom}(t)$ defined as

$$
\begin{aligned}
& V_{j}=\{v \in \rho: t(v)=(P, j) \text { and } \\
&\text { for every } \left.v^{\prime} \preceq v \text { we have } t(v)=\left(P^{\prime}, j^{\prime}\right) \text { with } j^{\prime} \leq j\right\} .
\end{aligned}
$$

The set $V_{j}$ with the prefix and lexicographic order is isomorphic to an $\omega$-forest $\tau$. Since $\rho$ is winning, so $\tau$ is well-founded. Let

$$
\begin{aligned}
\operatorname{rank}_{j}(t, \rho) & \stackrel{\text { def }}{=} \operatorname{rank}_{\mathrm{WF}}(\tau), \\
\operatorname{rank}(t, \rho) & \stackrel{\text { def }}{=} \sup _{v \in \rho, i \leq j^{\prime} \leq k, j^{\prime} \equiv 1(\bmod 2)} \operatorname{rank}_{j^{\prime}}\left(t \uparrow_{v}, \rho \uparrow_{v}\right), \\
\operatorname{rank}_{j}(t) & \stackrel{\text { def }}{=} \inf _{\sigma \text { winning for } \exists} \operatorname{rank}_{j}(t, \sigma) .
\end{aligned}
$$

Note that since $t \in \mathcal{W}_{i, k}$ and $\rho$ is winning, all the above ordinals are defined (i.e. not equal $\infty$ ). Now assume that $k$ is odd and $\alpha$ is a countable ordinal. Let

$$
\mathcal{W}_{i, k}^{\alpha} \stackrel{\text { def }}{=}\left\{t \in \mathcal{W}_{i, k}: \operatorname{rank}_{k}(t) \leq \alpha\right\} .
$$

Fact 1. For $i=0$ and $k=1$ the rank rank ${ }_{1}$ is a co-analytic rank in the meaning of [13]. 
Proof. Intuitively, the set $\mathcal{W}_{0,1}$ is equivalent to $\mathrm{WF}$ with preservation of ranks. Formally, this fact follows from the construction of a Borel derivative, see [13, Section 34.D and Theorem 34.10]. The construction is motivated by Definition 6.2 .14 in [19].

First note that the set $D_{0}$ of trees $t \in \mathcal{W}_{0,1}$ on which $\exists$ has a strategy that visits at most once priority 1 is a Borel subset of $\operatorname{Tr}_{0,1}$ : by Königs lemma it is enough to have longer and longer finite strategies visiting 1 at most once.

Now, the derivative $D$ inputs a tree $t \in \operatorname{Tr}_{0,1}$ and for every $w \in \operatorname{dom}(t)$ : if $t \uparrow_{w} \in D_{0}$ then replace whole subtree of $t$ under $w$ with a fixed tree $t_{0}$ labelled everywhere with $(\exists, 0)$.

Clearly, such a derivative is monotone with respect to priorities and players on the tree - it replaces some vertices of the form $(P, i)$ with $(\exists, 0)$. The fixpoint of the applications of $D$ to $t$ equals $t_{0}$ if and only if $t \in \mathcal{W}_{0,1}$. The number of applications of $D$ to $t$ until reaching $t_{0}$ (denoted $|t|_{D}$ in [13, Section 34.D]) is exactly $\operatorname{rank}_{1}(t)$. Therefore, $D$ is a Borel derivative and rank ${ }_{1}$ is a $\Pi_{1}^{1}$-rank.

Lemma 10. All the sets $\mathcal{W}_{i, k}^{\alpha}$ for $i<k$, with odd $k$ and $\alpha<\omega_{1}$ are measurable.

Proof. We prove in fact a stronger statement that for each $\alpha$ the set $\mathcal{W}_{i, k}^{\alpha}$ is on the $(k+1)$-th level of the $\mathcal{R}$-hierarchy. The proof is inductive on $\alpha$.

First observe that $\mathcal{W}_{i, k}^{0}$ is the set of trees where $\exists$ can win without visiting any node of priority $k$. Therefore, it continuously reduces to $\mathcal{W}_{i, k-1}$ by making the subtree under each node labelled by $k$ loosing for $\exists$. So $\mathcal{W}_{i, k}^{0}$ is on the $k$-th level of the $\mathcal{R}$-hierarchy.

The limit step is trivial, as it boils down to a countable union and each level of the $\mathcal{R}$-hierarchy is closed under countable unions and countable intersections - these operations can be simulated by the $\mathcal{R} \bigcup \circ \bigcap$ operation so the closure follows from Lemma 3.

For the successor step we introduce a derivative in the spirit of the above Fact 1. First let us fix a tree $t_{0}$ that is labelled everywhere with $(\exists, k-1)$. Since $k$ is odd, so $t_{0}$ is winning for $\exists$. Let $D_{0}$ be the set of all trees $t \in \operatorname{Tr}_{i, k}$ where $\exists$ can win without visiting any node of priority $k$ except the root that may potentially be labelled with $k$. Clearly, $D_{0} \sim_{W} \mathcal{W}_{i, k}^{0}$. Let $f$ be a function that inputs a tree $t \in \operatorname{Tr}_{i, k}$ and replaces every subtree $\left.t\right|_{w} \in D_{0}$ by $t_{0}$. The $f$-pre-image of each clopen set is a boolean combination of clopens and $D_{0}$, so it is a set on the $(k+1)$-th level of the $\mathcal{R}$ hierarchy.

Therefore, by Lemma 4 the $f$-pre-image of any set on the $(k+1)$-th level of the $\mathcal{R}$ hierarchy is also at this level. But

$$
f^{-1}\left(\mathcal{W}_{i, k}^{\alpha}\right)=\mathcal{W}_{i, k}^{\alpha+1}
$$

what finishes the induction step.

\section{C.2 The boundedness principle for $(0,1)$-index}

In this section we prove the first part of Theorem 6 , namely the continuity property for $i=0, k=1$. 
Assume, that there is given a measure $\mu$ such that $\mu\left(\mathcal{W}_{0,1}\right)>0$. We will show, that there exists $\alpha<\omega_{1}$ such that $\mu\left(\mathcal{W}_{0,1}^{\alpha}\right)=\mu\left(\mathcal{W}_{0,1}\right)$. Indeed, from the inner regularity of $\mu$ follows, that there exists a Borel set $G \subseteq \mathcal{W}_{0,1}$ such that $\mu(G)=\mu\left(\mathcal{W}_{0,1}\right)$. Recall that by Fact 1 , rank ${ }_{1}$ is a co-analytic rank on $\mathcal{W}_{0,1}$. Therefore, the Boundedness Principle ([13, Theorem 35.23]) guarantees, that $G$ is contained in $\mathcal{W}_{0,1}^{\alpha}$ for some $\alpha<\omega_{1}$. Hence

$$
\mu(G) \leq \mu\left(\mathcal{W}_{0,1}^{\alpha}\right) \leq \mu\left(\mathcal{W}_{0,1}\right)=\mu(G) .
$$

\section{C.3 Failure of the boundedness principle for higher ranks}

In this section we show that the method from Section C.2 does not generalize to higher indices. For a tree $t \in \operatorname{Tr}_{1,2}$ let us construct the dual tree $\bar{t} \in \operatorname{Tr}_{2,3}$ by replacing a label $(P, i)$ by $(\bar{P}, i+1)$. Clearly, $t \in \mathcal{W}_{1,2}$ if and only if $\bar{t} \notin \mathcal{W}_{2,3}$.

Now, consider the set of trees $K \subseteq \operatorname{Tr}_{1,3}$ of the form $f(t) \stackrel{\text { def }}{=}(\exists, 1)(t, \overline{(t} t)$ for $t \in \operatorname{Tr}_{1,2}$, as shown at Figure C.3.

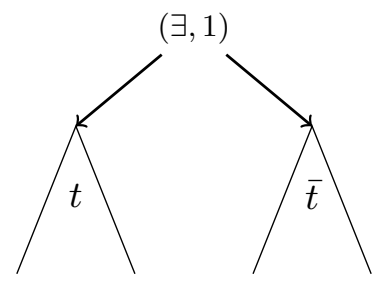

Fig. 9. The tree $f(t)$.

Note that $t \in K$ if it satisfies a closed constraint: for each vertex of the form $d v$ for $d \in\{0,1\}$ and $v \in\{0,1\}^{*}$, if $t(d v)=(P, i)$ then $t(\bar{d} v)=(\bar{P}, i+1)$. Therefore, $K \subseteq \operatorname{Tr}_{1,3}$ is a compact set.

Observe that for each $t \in \operatorname{Tr}_{1,2}$ either $t \in \mathcal{W}_{1,2}$ or $\bar{t} \in \mathcal{W}_{2,3}$ therefore

$$
K \subseteq \mathcal{W}_{1,3}
$$

Now consider $\operatorname{rank}_{3}(t)$ for trees $t \in K$. Observe that if $t \in \mathcal{W}_{1,2}$ then $\exists$ can win on $f(t)$ by moving to the left subtree (i.e. $t$ ). Such a strategy does not visit any 3 , so $\operatorname{rank}_{3}(t)=0$. Let $\bar{t} \in \mathcal{W}_{2,3}$ be a tree such that $\operatorname{rank}_{3}(\bar{t})=\eta$. Observe that in that case $t \notin \mathcal{W}_{1,2}$ so any winning strategy for $\exists$ in $f(t)$ has to move to $\bar{t}$. In particular, in that case

$$
\operatorname{rank}_{3}(f(t))=\operatorname{rank}_{3}(\bar{t})=\eta .
$$

Therefore, the set $K$ is a witness that Boundedness Principle does not hold: $K$ is a compact subset of $\mathcal{W}_{1,3}$ such that

$$
\sup _{t \in K} \operatorname{rank}_{3}(t)=\sup _{t \in \mathcal{W}_{2,3}} \operatorname{rank}_{3}(t)=\omega_{1} .
$$




\section{C.4 L. Harrington game}

In this section we present an adaptation of a game by L. Harrington (see [13, Theorem 36.20]). We start with a definition of a Harrington's game in a generic setup.

Let us fix a pair of Polish spaces $X, Y$. Assume that $\mu$ is a Borel measure on $X$. Our aim is to design a game for verifying if the projection to $X$ of a given subset of $X \times Y$ is of $\mu$-measure 0 .

Let $\iota: 2^{\omega} \rightarrow X \times Y$ be a surjective function onto $X \times Y$ such that for every open set $U \subseteq X \times Y$ the preimage $\iota^{-1}(U)$ is a Borel set. Such a function exists by [13, Theorem 16.5]. For technical reasons, it is convenient to represent elements of $X \times Y$ by bits that are encoded by such a function $\iota$. Let $I_{0}, I_{1}, \ldots$ be an enumeration of finite unions of basic open sets in $X$.

Let us fix a set $W \in X \times Y$, its projection $L=\pi_{X}(W)$, and $\varepsilon>0$. Consider the following perfect information infinite duration game $H \mathcal{G}(\varepsilon)$ : in round $i$

- Player II plays a number $z_{i}$,

- Player I plays a bit $b_{i}$.

Intuitively, Player I plays a sequence of bits that encode (via $\iota$ ) a pair $(x, y)$. His aim is to produce a sequence from $W$. Player II plays indices of sets $I_{z_{0}}, I_{z_{1}}, \ldots$ His aim is to present a small cover of $L$.

Formally, the winning condition for Player I is the following:

1. either for some $i$ we have

$$
\mu\left(I_{z_{i}}\right)>\varepsilon \cdot 2^{-2 i},
$$

2. or the pair $(x, y)=\iota\left(b_{0} b_{1} \cdots\right)$ satisfies

$$
(x, y) \in W \quad \text { and } \quad x \notin \bigcup_{i \in \mathbb{N}} I_{z_{i}}
$$

Note that the descriptive complexity of the winning condition depends mostly on the set $W$, i.e. if $W \in \boldsymbol{\Pi}_{1}^{1}$ then the whole winning condition is also $\boldsymbol{\Pi}_{1}^{1}$.

The following proposition expresses the crucial property of the game $H \mathcal{G}$.

Proposition 2. Let $W \subseteq X \times Y$ be any set in a product of two spaces and let $L=\pi_{X}(W)$. The following conditions are equivalent:

- $L$ is of $\mu$-measure 0 ,

- for every $\varepsilon>0$ Player II has a winning strategy in $H \mathcal{G}(\varepsilon)$.

Proof. First assume that $L$ is of $\mu$-measure 0 . Take any $\varepsilon>0$ and find a cover of $L$ by a sequence of basic open sets $J_{0}, J_{1}, \ldots$ of measures summing up to $\varepsilon$. We can inductively find a sequence of indices $z_{0}, z_{1}, \ldots$ such that

$$
\bigcup_{i \in \mathbb{N}} I_{z_{i}}=\bigcup_{i \in \mathbb{N}} J_{i} \supseteq L \quad \text { and } \quad \forall_{i \in \mathbb{N}} \mu\left(I_{z_{i}}\right) \leq \varepsilon \cdot 2^{-2 i} .
$$


Consider the strategy of Player II that does not depend on the bits played by Player I and successively plays $z_{0}, z_{1}, \ldots$. This strategy is winning because of $(8)$.

Now assume that Player II has a winning strategy in all the games $H \mathcal{G}(\varepsilon)$. Take any $\varepsilon>0$ and let $\sigma_{\mathrm{II}}: 2^{*} \rightarrow \mathbb{N}$ be a winning strategy of Player II in $H \mathcal{G}(\varepsilon)$. Consider the set

$$
U=\bigcup_{w \in 2^{*}} I_{\sigma_{\mathrm{II}}(w)} .
$$

Using Condition 1 we know:

$$
\begin{aligned}
\forall_{w \in 2^{*}} \mu\left(I_{\sigma_{\mathrm{II}}(w)}\right) & \leq \varepsilon \cdot 2^{-2|w|} \\
\forall_{n \in \mathbb{N}} \bigcup_{w \in 2^{n}} \mu\left(I_{\sigma_{\mathrm{II}}(w)}\right) & \leq \varepsilon \cdot 2^{-n} \\
\sum_{w \in 2^{*}} \mu\left(I_{\sigma_{\mathrm{II}}(w)}\right) & \leq \varepsilon
\end{aligned}
$$

What remains is to show that $L \subseteq U$. Assume contrary that there exists an element $(x, y) \in W$ such that $x \notin U$. Consider the strategy $\sigma_{\mathrm{I}}$ of Player I that plays bits $b_{0}, b_{1}, \ldots$ such that $\iota\left(b_{0} b_{1} \cdots\right)=(x, y)$. Let $\alpha=\left(z_{0}, b_{0}, z_{1}, b_{1}, \ldots\right)$ be the play resulting from strategies $\sigma_{\mathrm{I}}$ and $\sigma_{\mathrm{II}}$. Observe that this play satisfies Condition 2: $(x, y) \in W$ and $x \notin U \supseteq \bigcup_{i \in \mathbb{N}} I_{z_{i}}$. Therefore, $\alpha$ is winning for Player I what contradicts that $\sigma_{\text {II }}$ is winning for Player II.

Now we can consider what are the consequences of existence of a winning strategy for Player I.

Lemma 11. If for some $\varepsilon>0$ Player I has a winning strategy in $H \mathcal{G}(\varepsilon)$ then there exists a $\Sigma_{1}^{1}$ set $S \subseteq W$ such that

$$
\mu\left(\pi_{X}(S)\right)>0 .
$$

Proof. Let $\sigma_{\mathrm{I}}$ be a winning strategy for Player I. Let

$$
\begin{aligned}
S=\left\{\iota\left(b_{0} b_{1} \cdots\right): \exists_{\left(z_{0}, z_{1}, \ldots\right)}\right. & \forall_{i \in \mathbb{N}} \mu\left(I_{z_{i}}\right) \leq \varepsilon \cdot 2^{-2 i} \text { and } \\
& \left.\left(b_{0}, b_{1}, \ldots\right) \text { is the response of } \sigma_{\mathrm{I}} \text { to }\left(z_{0}, z_{1}, \ldots\right)\right\} .
\end{aligned}
$$

Clearly $S \in \boldsymbol{\Sigma}_{1}^{1}(X \times Y)$. Since $\sigma_{\mathrm{I}}$ is a winning strategy, so by Condition 2 we know that $S \subseteq W$. Assume that $\mu\left(\pi_{X}(S)\right)=0$. Similarly as in the proof of Proposition 2 we can construct a strategy $\sigma_{\text {II }}$ of Player II that enumerates a small cover of $\pi_{X}(S)$. Let $\left(z_{0}, b_{0}, z_{1}, b_{1}, \ldots\right)$ be the play resulting from $\sigma_{\mathrm{I}}$ and $\sigma_{r} I I$. By the definition we know that $(x, y)=\iota\left(b_{0} b_{1} \cdots\right) \in S$, therefore $x \in \bigcup_{i \in \mathbb{N}} I_{z_{i}}$ so this play is winning for Player II by Condition 2, a contradiction.

\section{C.5 Proof of the second part of Theorem 6}

Theorem 7 ( $\Sigma_{1}^{1}$ determinacy). Fix an index $(i, k)$ and let $\mu$ be a Borel measure on $\operatorname{Tr}_{i, k}$. Then

$$
\sup _{\alpha<\omega_{1}} \mu\left(\mathcal{W}_{i, k}^{\alpha}\right)=\mu\left(\mathcal{W}_{i, k}\right) .
$$


Note that all the sets in the above statement are measurable by Theorem 1 and Lemma 10. The rest of this section is devoted to Theorem 7.

Lemma 12. It is enough to prove Theorem 7 assuming that for all $\alpha<\omega_{1}$ we have

$$
\mu\left(\mathcal{W}_{i, k}^{\alpha}\right)=0
$$

Proof. Let $x$ be the supremum of the sequence $\mu\left(\mathcal{W}_{i, k}^{\alpha}\right)$ for $\alpha<\omega_{1}$. Since the length of this sequence is uncountable and the values are non-decreasing, there exists $\alpha<\omega_{1}$ such that

$$
x=\mu\left(\mathcal{W}_{i, k}^{\alpha}\right) .
$$

If the measure of the complement of $\mathcal{W}_{i, k}^{\alpha}$ is 0 then $\mu\left(\mathcal{W}_{i, k}\right)=x$ and the thesis holds. Consider a new measure $\mu^{\prime}$ on the same family of sets, defined as

$$
\mu^{\prime}(A)=\mu\left(A \backslash \mathcal{W}_{i, k}^{\alpha}\right) .
$$

Observe that since $\mathcal{W}_{i, k}^{\alpha}$ is measurable (see Fact 10), the measure $\mu^{\prime}$ is a Borel measure on $\operatorname{Tr}_{i, k}$. By our assumption, the $\mu^{\prime}$-measure of all the sets $\mathcal{W}_{i, k}^{\alpha}$ is 0 and it remains to prove that the $\mu^{\prime}$-measure of $\mathcal{W}_{i, k}$ is also 0.

Let us assume that $\mathcal{W}_{i, k}$ is not of $\mu$-measure 0 . Our aim is to show that for some $\alpha<\omega_{1}$ the set $\mathcal{W}_{i, k}^{\alpha}$ is also not of $\mu$-measure 0 . Our plan is to construct a special Harrington game. Then, by $\boldsymbol{\Sigma}_{1}^{1}$-determinacy and Lemma 11 we will obtain an analytic set $S \subseteq W$. By the Boundedness Principle, this $S$ will be bounded with respect to an adequate rank on $W$ and its projection to $X$ will be contained in some $\mathcal{W}_{i, k}^{\alpha}$.

Let us fix $X$ to be the space of all game trees with priorities $(i, k): X=\operatorname{Tr}_{i, k}$. The space $Y$ will be the product of three spaces:

- Runs - the space of strategies of $\exists$ on trees in $\operatorname{Tr}_{i, k}$,

- $\operatorname{Tr}(\omega)$ - the space of $\omega$-trees,

$-\mathcal{J}=\left(\omega^{*}\right)^{2^{*}}$ - the space of functions from $2^{*}$ (i.e. the domains of $t \in \operatorname{Tr}_{i, k}$ ) to $\omega^{*}$ (i.e. the vertices of trees in $\operatorname{Tr}(\omega)$ ).

We will denote an element of $X \times Y$ by $(t, \rho, \tau, \eta)$ with $t \in \operatorname{Tr}_{i, k}, \rho \in$ Runs, $\tau \in \operatorname{Tr}(\omega)$, and $\eta \in \mathcal{J}$. Now we can define the winning set $W \subseteq X \times Y$. The crucial requirement in this definition will be that $\tau$ is a well-founded tree (i.e. $\tau \in \mathrm{WF}$ ). Also, we will need the following definition:

Definition 9. Let $t \in \operatorname{Tr}_{i, k}$ and $u \prec v$ be a pair of vertices of $t$. We say that $u, v$ are odd-dominating if both of them have the same odd priority in $t$ and there is no higher priority between them in $t$. Formally:

$t(u)=\left(P_{u}, n\right)$ for a player $P_{u}$ and an odd priority $n$, $t(v)=\left(P_{v}, n\right)$ for the same priority $n$,

for every $w$ such that $u \prec w \prec v$ the priority of $w$ in $t$ is at most $n$.

Let $W \subseteq X \times Y$ contain those tuples $(t, \rho, \tau, \eta)$ that satisfy the following conditions: 
1. $\tau \in \mathrm{WF}$,

2. $\rho$ is a strategy for Eve on $t$,

3. for every pair of vertices $u \prec v$ that are accessible by $\rho$, if $u, v$ are odddominating then

$$
\eta(u) \prec \eta(v) .
$$

Clearly, all the requirements of this definition, except the first one, are Borel. The first condition is $\boldsymbol{\Pi}_{1}^{1}$.

Lemma 13. The projection of $W$ onto $\operatorname{Tr}_{i, k}$ is $\mathcal{W}_{i, k}$. Additionally, if $(t, \rho, \tau, \eta) \in$ $W$ then

$$
\operatorname{rank}_{k}(t) \leq \operatorname{rank}_{\mathrm{WF}}(\tau) .
$$

Proof. First observe that if $(t, \rho, \tau, \eta) \in W$ then $\rho$ is a winning strategy of $\exists$ on $t$. Assume contrary that there exists a branch $\pi \in 2^{\omega}$ of $\rho$ such that the limes superior $j$ of priorities of $t$ on $\pi$ is odd. Therefore, there is a sequence $u_{0} \prec u_{1} \prec \ldots$ of vertices of $t$ on $\pi$ such that for every $i$ the pair $u_{i}, u_{i+1}$ is odddominating. Therefore, by Condition $3 \eta\left(u_{0}\right) \prec \eta\left(u_{1}\right) \prec \ldots$ is a witness that $\tau$ contains an infinite branch, so $\tau \notin \mathrm{WF}$. A contradiction.

Now we prove Equation (9). Observe that $\operatorname{rank}_{k}(t, \rho) \leq \operatorname{rank}(t, \rho)$. Let $X$ be the set of vertices of $\rho$ of priority $k$. We can see $(X, \preceq)$ an infinitely branching tree. Since $k$ is the highest priority and $k$ is odd, so every pair of vertices $u \prec v$ in $X$ is odd-dominating. Therefore, $\eta \uparrow_{X}$ is an embedding of $(X, \preceq)$ to $\tau$, so

$$
\operatorname{rank}_{k}(t, \rho) \leq \operatorname{rank}_{\mathrm{WF}}(\tau) .
$$

Not take a tree $t \in \mathcal{W}_{i, k}$. Let $\rho$ be a winning strategy for $\exists$ on $t$. We want to find $\tau$, and $\eta$ such that $(t, \rho, \tau, \eta) \in W$. Let

$$
\alpha=\operatorname{rank}(t, \rho) .
$$

Since $\rho$ is a winning strategy, so $\alpha<\omega_{1}$. Let $\tau \in \mathrm{WF}$ be an $\omega$-tree such that $\operatorname{rank}_{\mathrm{WF}}(\tau)>\alpha$. Now, using (10) we inductively construct $\eta$ satisfying Condition 3. The induction invariant is that for every $v \in \rho$ with $t(v)=\left(P_{v}, n_{v}\right)$ and every odd $j \leq n_{v}$ we have

$$
\operatorname{rank}_{j}\left(t \uparrow_{v}\right)<\operatorname{rank}_{\mathrm{WF}}\left(\tau \uparrow_{\eta(v)}\right) .
$$

For $\eta$ constructed this way we clearly have $(t, \rho, \tau, \eta) \in W$.

Now we consider the Harrington game $H \mathcal{G}(\varepsilon)$ with the winning set $W \subseteq$ $X \times Y$. The above lemma says that $L=\pi_{X}(W)$ equals $\mathcal{W}_{i, k}$. By our assumption that $\mathcal{W}_{i, k}$ is not of $\mu$-measure 0 , Proposition 2 implies that Player I does not have a winning strategy in $H \mathcal{G}(\varepsilon)$ for some $\varepsilon>0$. By $\Sigma_{1}^{1}$-determinacy it means that Player II has a winning strategy in $H \mathcal{G}(\varepsilon)$. By Lemma 11, there is an analytic set $S \subseteq W$ such that

$$
\mu\left(\pi_{X}(S)\right)>0 .
$$


Observe that the set of trees $\tau \in \operatorname{Tr}(\omega)$ that appear in $S$ is also analytic - it is the projection of $S$ onto $\operatorname{Tr}(\omega)$. Therefore, by the Boundedness Principle (see [13, Theorem 35.23]) there is an ordinal $\alpha<\omega_{1}$ such that for every $(t, \rho, \tau, \eta) \in S$ we have $\operatorname{rank}_{\mathrm{WF}} \tau<\alpha$. By Lemma 13, for every $(t, \rho, \tau, \eta) \in S$ there holds

$$
\operatorname{rank}_{k}(t) \leq \operatorname{rank}_{\mathrm{WF}}(\tau)<\alpha .
$$

Therefore, the projection $\pi_{X}(S)$ is contained in $\mathcal{W}_{i, k}^{\alpha}$ and

$$
\mu\left(\mathcal{W}_{i, k}^{\alpha}\right) \geq \mu\left(\pi_{X}(S)\right)>0 .
$$

